Annales Geophysicae (2003) 21: 1315-1330 (C) European Geosciences Union 2003

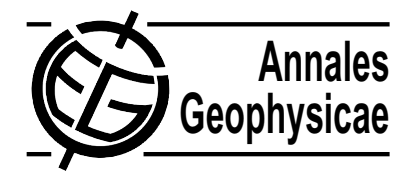

\title{
Imprints from the solar cycle on the helium atom and helium pickup ion distributions
}

\author{
D. Ruciński ${ }^{1}{ }^{\dagger}$, M. Bzowski ${ }^{2}$, and H. J. Fahr ${ }^{2}$ \\ ${ }^{1}$ Space Research Centre PAS, Bartycka 18A, 00-716 Warsaw, Poland \\ ${ }^{2}$ Institut für Astrophysik und Extraterrestrische Forschung der Universität Bonn, Auf dem Hügel 71, D-53121 Bonn, Germany \\ ${ }^{\dagger}$ deceased in February 2002
}

Received: 17 September 2002 - Revised: 31 January 2003 - Accepted: 4 March 2003

\begin{abstract}
Neutral interstellar helium atoms penetrate into the solar system almost unaffected by gas-plasma interactions in the heliospheric interface region, and thus can be considered as carriers of original information on the basic parameters (like density, temperature, bulk velocity) of the Very Local Interstellar Medium (VLISM). Such information can nowadays be derived from analysis of data obtained from different experimental methods: in situ measurements of He atoms (Ulysses), observations of the solar backscattered $\mathrm{He} 584 \AA$ radiation (EUVE), in situ measurements of $\mathrm{He}^{+}$ pickup ions (AMPTE, Ulysses, Wind, SOHO, ACE). In view of the current coordinated international ISSI campaign devoted to the study of the helium focusing cone structure and its evolution, we analyze expected variations of neutral $\mathrm{He}$ density, of $\mathrm{He}^{+}$pickup fluxes and of their phase space distributions at various phases of the solar activity cycle based on a realistic time-dependent modelling of the neutral helium and $\mathrm{He}^{+}$pickup ion distributions, which reflect solar cycleinduced variations of the photoionization rate. We show that the neutral helium density values are generally anticorrelated with the solar activity phase and in extreme cases (near the downwind axis) the maximum-to-minimum density ratio may even exceed factors of $\sim 3$ at $1 \mathrm{AU}$. We also demonstrate that in the upwind hemisphere (at $1 \mathrm{AU}$ and beyond) the $\mathrm{He}^{+}$fluxes are correlated with the solar cycle activity, whereas on the downwind side the maximum of the expected flux up to distances of $\sim 3 \mathrm{AU}$ occurs around solar minimum epoch, and only further away does the correlation with solar activity become positive. Finally, we present the response of the phase space distribution spectra of $\mathrm{He}^{+}$pickup ions (in the solar wind frame) for different epochs of the solar cycle and heliocentric distances from 1 to $5 \mathrm{AU}$ covering the range of Ulysses, Wind and ACE observations.
\end{abstract}

Key words. Solar physics, astrophysics and astronomy (ultraviolet emissions) - Space plasma physics (ionization processes; numerical simulation studies)

Correspondence to: M. Bzowski (bzowski@cbk.waw.pl)

\section{Introduction}

Interstellar helium is the second most abundant element in the Local Interstellar Cloud (LIC) and since it does not interact with the heliospheric interface, it allows for the determination of the physical state of interstellar gas in the immediate solar neighbourhood. Hence, the information obtained from measurements of the interstellar helium gas has been used for quite some time as a valuable reference to study other interstellar elements inside the heliosphere and some element-specific processes (like charge exchange) to which these are subjected in the heliospheric interface. The LIC helium gas has been studied by numerous space-borne observations of the interplanetary glow in the He-584 $\AA$ line, starting with the Nike sounding rocket observations by Paresce et al. (1974a,b) and STP satellite observations by Weller and Meier (1974), followed by second generation experiments (Weller and Meier, 1981; Burgin et al., 1983; Dalaudier et al., 1984; Chassefière et al., 1986) and presently by the most recent EUVE and SOHO satellite observations, respectively by Flynn et al. (1998) and Michels et al. (2002). The first proposal to observe the EUV-resonance glow of the derivative products of the LIC helium, i.e. of $\mathrm{He}^{+}$pickup ions excited by the solar $304 \AA$ emission line, goes back to the early work by Paresce et al. (1981) and a later work by Fahr et al. (1998) but unfortunately such observations have not been carried out yet.

There are plenty of direct observations of the LIC helium atoms presence in the heliosphere and of the derivative helium ions in the interplanetary space. Since 1990, the GAS experiment on board of ULYSSES has been registering in situ neutral interstellar helium atoms in the interplanetary space. From these observations, Witte et al. (1996; 2003), derived LIC helium properties with great accuracy. The first direct observation of the derivative ion population co-moving with the solar wind, i.e. of the interplanetary $\mathrm{He}^{+}$pickup ions (PUIs), was performed in 1985 by the AMPTE/ IRM mission (Möbius et al., 1985). More recently, extremely valuable observations of heliospheric $\mathrm{He}^{+}$and $\mathrm{He}^{++}$pickup ions 
with detailed spectral information on relevant kinetic transport processes were performed by the Ulysses/SWICS experiment (Gloeckler et al., 1994; Gloeckler, 1996) and by SOHO (Möbius et al., 1995, 1996).

Early UV observations (Weller and Meier, 1974, 1979, 1981; Paresce et al., 1974a,b; Freeman et al., 1976, 1979; Dalaudier et al., 1984; Burgin et al., 1983; Kurt et al., 1984a,b; Chassefière et al., 1986), supported by appropriate modelling (Blum et al., 1975; Fahr et al., 1976, 1987), led to the conclusion that the interstellar helium at the upwind boundary of the heliosphere had a bulk velocity of about $V=27 \mathrm{~km} / \mathrm{s}$ and a temperature of about $T=15000 \mathrm{~K}$, surprisingly different from values derived for the velocity and temperature of neutral hydrogen in the Very Local InterStellar Medium (correspondingly, $20 \mathrm{~km} / \mathrm{s}$ and $8000 \mathrm{~K}$, see Bertaux et al., 1985). It was soon realized that most probably the velocity vectors and kinetic temperatures of these two elements in the interstellar space were identical but a discrepancy of these quantities at the termination shock of the solar wind should be expected. This is due to elementselective influence of the heliospheric interface, which via charge exchange reactions couples to hydrogen and some other elements but not to helium (see Osterbart and Fahr, 1992; Ruciński et al., 1993; Fahr et al., 1993) and hence the hydrogen characteristics at the termination shock should be modified but those of helium should stay intact. Direct measurements of interstellar helium by the Ulysses/GAS experiment (Witte et al., 1996) basically confirmed the magnitude and direction of the bulk velocity vector (recent values $26.4 \mathrm{~km} / \mathrm{s}$ for ecliptic longitude and latitude of the upwind direction equal to $254.7^{\circ}$ and $5.6^{\circ}$, Witte et al., 2003) but showed at the same time that the temperature is much lower $(6680 \mathrm{~K})$ than the apparent hydrogen temperature at the termination shock $(12000 \mathrm{~K}$ for the bulk speed of $\sim 22 \mathrm{~km} / \mathrm{s}$, Lallement, 1999; Costa et al., 1999). The recent Ulysses results on helium are in excellent agreement with astrophysical measurements: $25.7 \mathrm{~km} / \mathrm{s}, 7000 \mathrm{~K}, 254.9^{\circ}, 7.8^{\circ}$ (Lallement and Bertin, 1992).

The early determinations of helium interstellar parameters were mainly based on measurements of the full width at half maximum of the helium cone glow, which is proportional to the quantity $\sqrt{T} / V$ (see, e.g. Feldman et al., 1972; Chassefière et al., 1988), as predicted by the modified cold (Blum et al., 1975) or the hot stationary model (Fahr, 1971, 1979; Wu and Judge, 1979) of helium distribution. Since, however, the results of such models are valid only for a time-invariant helium ionization rate, they can provide only a qualitative insight into the actual helium density distribution. In reality, the ionization rate is variable in time and this variability has a significant effect. This was realized early on by Ruciński (1985, Ph.D. thesis) and Fahr et al. (1987). Time-dependent models were also seen as a necessary prerequisite for the interpretation of $\mathrm{He}^{+}$PUI measurements (Ruciński and Fahr, 1991). As we will show here, time-dependent modeling is an essential tool for the interpretation of VLISM helium data and for supporting the analysis efforts for the current coordinated international ISSI campaign of various (optical, pickup ion, neutral atom) He-related measurements, including Ulysses, SOHO, Wind, ACE, EUVE, Nozomi, Geotail, and IMAGE missions. In the present paper we will study qualitatively various time-dependent effects in helium of interstellar origin in the inner heliosphere and in its derivative $\mathrm{He}^{+}$pickup population in the solar wind. We will investigate the magnitude of deviations of relevant quantities from mean values during the solar cycle to find the regions where timedependent effects are significant. We do not aim, however, to provide quantitative prediction of helium density and pickup flux for the needs of actual analysis of data from space experiments. In our opinion, this goal can only be realized when more accurate data on the helium photoionization rate become available from direct measurements or at least from reliable proxies. Furthermore, electron impact ionization must be consistently taken into account, and both time-dependent and 3-D effects have not been adequately derived yet.

\section{Modelling of interplanetary helium}

2.1 Definitions of distribution function, density, pickup production rate, and pickup flux

In our modelling of the interstellar helium distribution in the inner heliosphere we adopted the scheme developed by Ruciński (1985, Ph.D. thesis) and Fahr et al. (1987): The distribution function $f_{\infty}(\boldsymbol{v})$ of the unperturbed gas (far away from the Sun, $r>100 \mathrm{AU}$ ) is represented by a Maxwellian "shifted" by its flow vector $v_{B}$ :

$f_{\infty}(\boldsymbol{v})=n_{\infty}\left(\frac{m}{2 \pi k T_{\infty}}\right)^{\frac{3}{2}} \exp \left[-\frac{m\left(\boldsymbol{v}-\boldsymbol{v}_{\mathrm{B}}\right)^{2}}{2 k T_{\infty}}\right]$.

Here, $n_{\infty}$ and $T_{\infty}$ denote the unperturbed VLISM helium density and temperature. Since the solar radiation pressure is negligible for helium atoms, they feel only the stationary solar gravitational force. They are subjected, however, to nonstationary spherically symmetric (as we adopt here) ionization losses, whose rates decrease with heliocentric distance as $1 / r^{2}$. This assumption is sufficiently well fulfilled outside $1 \mathrm{AU}$; closer to the Sun the ionization by electron impact becomes increasingly significant (Ruciński et al., 1996), but this effect was excluded from the analysis presented here.

The local helium density $n_{\mathrm{He}}(r, \theta, t)$ in a space point determined by the heliocentric distance $r$, an offset angle $\theta$ from the upwind direction, and a time $t$ is calculated by integration of the local distribution function of the gas $f_{\mathrm{LOC}}$ in the 3 -D velocity space

$n_{\mathrm{He}}(r, \theta, t)=\int f_{\mathrm{LOC}}(r, \theta, t, v) d^{3} v$

and the local distribution function is a product of the distribution function of the unperturbed gas and of the extinction function $E(r, \theta, \boldsymbol{v}, t)$ :

$f_{\mathrm{LOC}}(r, \theta, t, \boldsymbol{v})=f_{\infty}\left(\boldsymbol{v}_{0}(\boldsymbol{v}, r, \theta)\right) E(r, \theta, \boldsymbol{v}, t)$. 
The unperturbed distribution function must be evaluated for a velocity vector $\boldsymbol{v}_{0}$ which is ballistically connected to the local velocity vector $\boldsymbol{v}$ at $(r, \theta)$ and the atom extinction function $E$ is just the probability of survival of the test atom on the way from the unperturbed region to the local point during its travel in the time-dependent, spherically symmetric ionization field (see also Ruciński and Bzowski, 1995):

$$
\begin{aligned}
E(r, \theta, \boldsymbol{v}, t) & \\
\quad & =\exp \left[-\int_{t^{\prime}=t}^{\infty} \beta_{\text {loss }}\left(r_{\mathrm{E}}, t^{\prime}\right)\left(\frac{r_{\mathrm{E}}}{r^{\prime}\left(r, \theta, \boldsymbol{v}, t^{\prime}\right)}\right)^{2} d t^{\prime}\right] .
\end{aligned}
$$

In this equation, $\beta_{\text {loss }}\left(r_{\mathrm{E}}, t^{\prime}\right)$ is the helium ionization (loss) rate at time $t^{\prime}$ and heliocentric distance $r_{\mathrm{E}}$, and $r^{\prime}\left(r, \theta, \boldsymbol{v}, t^{\prime}\right)$ is the heliocentric distance of the test atom during its flight between the unperturbed region and the local point $(r, t)$ at time $t^{\prime}$.

The local production rate $\sigma(r, \theta, t)$ of $\mathrm{He}^{+}$pickup ions is equal to

$\sigma(r, \theta, t)=n_{\mathrm{He}}(r, \theta, t) \beta_{\text {prod }}(r, \theta, t)$,

where $\beta_{\text {prod }}(r, \theta, t)$ is the combined instantaneous rate of all processes leading to the creation of $\mathrm{He}^{+} \mathrm{PUI}$; in this analysis only photoionization by solar EUV radiation was taken into account. The total flux of pickup ions was calculated with a formula similar to that proposed by Vasyliunas and Siscoe (1976):

$$
\begin{aligned}
& F_{\mathrm{He}^{+}}(r, \theta, t)=\frac{1}{r^{2}} \int_{r_{0}}^{r} \sigma\left(r^{\prime}, \theta, t\right) r^{\prime 2} d r^{\prime} \\
& =\left(\frac{r_{\mathrm{E}}}{r}\right)^{2} \int_{r_{0}}^{r} n_{\mathrm{He}}\left(r^{\prime}, \theta, t\right) \beta_{\text {prod }}\left(r_{\mathrm{E}}, \theta, t\right) d r^{\prime},
\end{aligned}
$$

where formally $r_{0}$ is the solar radius and quasi-stationarity conditions are assumed:

$$
\frac{n_{\mathrm{He}}(r, \theta, t)}{\left(\frac{\partial n_{\mathrm{He}}(r, \theta, t)}{\partial t}\right)} \gg \frac{r-r_{0}}{V} \text {. }
$$

For observations at $1 \mathrm{AU}$ the critical time defined at the righthand side of Eq. 7 is equal to $\sim 4$ days in the slow solar wind $(V=400 \mathrm{~km} / \mathrm{s})$ and $\sim 2$ days in the fast solar wind $(V=$ $800 \mathrm{~km} / \mathrm{s}$ ). For observations at $5 \mathrm{AU}$ this time increases 5 fold, that is to 20 or 10 days, and becomes comparable with the solar rotation period which is a good representation for the time scale of modulation of the $\mathrm{He}^{+}$PUI production rate. In such case, when the variability rate of the production rate is high, a more general form of Equation 6 should be used:

$$
F_{\mathrm{He}^{+}}(r, \theta, t)=\left(\frac{r_{\mathrm{E}}}{r}\right)^{2} \int_{r_{0}}^{r} n_{\mathrm{He}}\left(r^{\prime}, \theta, t^{\prime}\right) \beta_{\text {prod }}\left(r_{\mathrm{E}}, \theta, t^{\prime}\right) d r^{\prime},(8)
$$

where $t^{\prime}=t-\frac{r-r^{\prime}}{V}$ (we owe this fragment to one of the anonymous referees). In our modelling we considered only

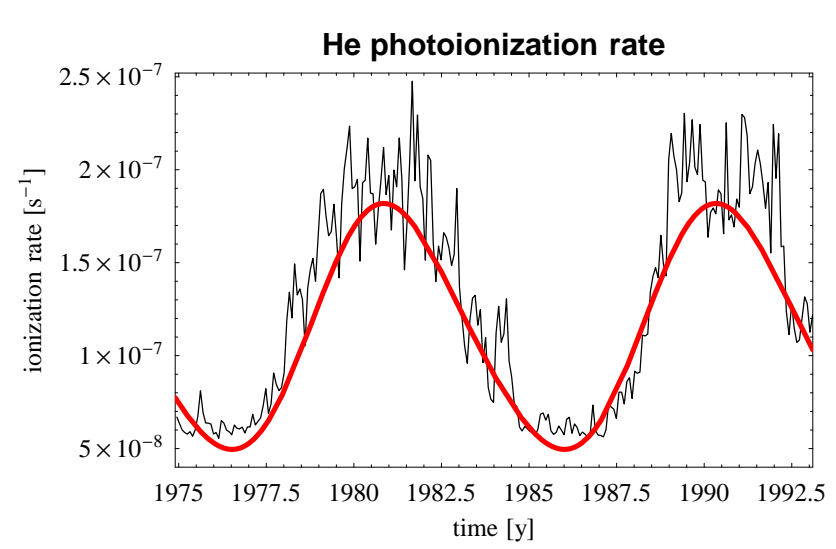

Fig. 1. Evolution of helium photoionization rate in time used in this study (red) shown in comparison to the actual instantaneous ionization rate inferred from the $10.7 \mathrm{~cm}$ proxy.

slowly varying PUI production processes and assumed that they are spherically symmetric, i.e. that $\beta_{\text {prod }}\left(r_{\mathrm{E}}, \theta, t\right)$ in fact does not depend on $\theta$.

In the calculations of gas density and PUI fluxes we used the following values of VLISM helium parameters: $n_{\mathrm{He}, \infty}=$ $0.015 \mathrm{~cm}^{-3} ; T_{\mathrm{He}, \infty}=7000 \mathrm{~K} ; v_{B}=25.3 \mathrm{~km} \mathrm{~s}^{-1}$; $\beta_{\mathrm{He}}\left(r_{E}, t\right)=$ ionization rate at $1 \mathrm{AU}$ - varying in time; the variations are considered as those during cycles 21 and 22, averaged over solar rotation period; $\mu=0$, i.e. the ratio of the solar radiation pressure to the solar gravity vanishes.

\subsection{Helium ionization rate used in this study}

Ionization processes play a dual role for helium: (1) their action accumulated for several months/years shapes a specific pattern of density distribution in the inner heliosphere; their rate $\beta_{\text {loss }}(t)$ in this context can be described by a slowly variable function shown in Fig. 1; (2) the instantaneous rates $\beta_{\text {prod }}$ (changing within days/weeks and not necessarily spherically symmetric) determine the actual production and resulting fluxes of relevant pickup ion species observed by spacecraft inside $\sim 5-10 \mathrm{AU}$.

The values of the long-term average $\beta_{\text {loss }}$ of the He loss rate and the instantaneous $\mathrm{He}^{+}$PUI production rate $\beta_{\text {prod }}$ differ because of the following reasons: (a) the long-term average rate represented by $\beta_{\text {loss }}$ is usually different than the instantaneous value of ionization rate at the time just preceding the pickup ion measurement; (b) not all ionization processes contributing to the ionization (loss) of neutrals may, in general, contribute to the production of particular pickup ion species. A more detailed discussion of these issues is provided by Ruciński et al. $(1996,1998)$.

The ionization rate variation used in this study was based on the $10.7 \mathrm{~cm}$ radio flux proxy. Since our aim was to indicate the amplitude of relevant variabilities in neutral helium density and in $\mathrm{He}^{+}$pickup flux and distribution function, rather than to perform an up-to-date precise modelling of these quantities, we use the variation of the ionization rate 


\section{Mean density/Density for mean ionization}

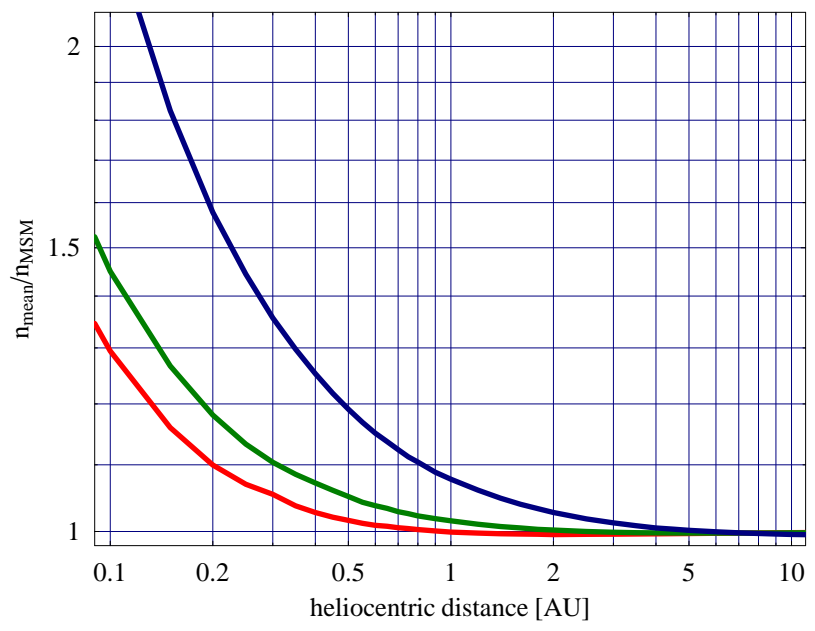

Fig. 2. Ratios of helium densities averaged over solar cycle to mean stationary densities, evaluated using the stationary model with the ionization rate equal to the mean value over the solar cycle. Red corresponds to the upwind, green to crosswind, blue to downwind axes.

based on the $10.7 \mathrm{~cm}$ radio flux proxy, adopted after Ruciński et al. (1996). The long-term rate $\beta_{\text {loss }}$ adopted here does not differ significantly from the actual photoionization rate measured by McMullin et al. (in preparation); the differences occur mainly during solar maximum, when the $10.7 \mathrm{~cm}$ proxy seems to slightly overestimate the actual helium photoionization rate. The adopted photoionization rate is shown in Fig. 1, along with the instantaneous radiation output inferred from the $10.7 \mathrm{~cm}$ proxy.

\subsection{Evolution of helium density during solar cycle}

Helium density averaged over the solar cycle is approximately equal to the density calculated with the use of the hot stationary model, with the ionization rate equal to the mean value of the ionization rate, except inside $\sim 2 \mathrm{AU}$ in the cone and $\sim 1$ AU elsewhere. The calculations for the mean ionization rate were performed using $\beta_{0}=1.13 \cdot 10^{-7} \mathrm{~s}^{-1}$, which is the mean value of the adopted ionization function $\beta(t)$, presented in Fig. 1. Throughout this paper, we will refer to these results as the mean stationary model (MSM). The mean density during the solar cycle was calculated as the arithmetic mean of the time dependent densities from 1976 untill 1994, sampled every year, and in the paper will be referred to as the mean density. The ratios of the mean density to the MSM density along the upwind, crosswind, and downwind axes are presented in Fig. 2.

The long-term departures of interstellar helium density from the solar-cycle mean, caused by solar cycle variations of the photoionization rate $\beta_{\text {loss }}(t)$, are only pronounced in the inner heliosphere. They are the strongest close to the Sun and drop down to a $10 \%$ level at about $20 \mathrm{AU}$ in the cone region and at about $4 \mathrm{AU}$ in the upwind and crosswind directions. This effect is clearly seen in Fig. 3 and Figs. 4 through 6 . In the first of these figures, the upper left panel shows the helium density $n_{\text {mean }}(r, \theta)$ as a function of heliocentric distance for the upwind, crosswind and downwind directions, averaged between 1976 and 1994. The density is shown as a fraction of the gas density at infinity. This panel provides reference for the remaining three panels of Fig. 3, where the ratios of the actual helium density $n(r, \theta, t)$ to the mean are presented as a function of the heliocentric distance for the upwind, crosswind and downwind directions: $\frac{n(r, \theta, t)}{n_{\text {mean }}(r, \theta)}$ for $\theta$ equal to $0^{\circ}, 90^{\circ}$ and $180^{\circ}$, respectively.

The extreme upper and lower values in the upwind, crosswind and downwind panels of Fig. 3 can be regarded as boundaries of the variability envelope of interstellar helium for the relevant directions in the heliosphere.

The departures of density from the mean value increase rapidly with the decrease in heliocentric distance, as it is shown in this figure and even more clearly in Figs. 4 through 6. At $1 \mathrm{AU}$ they exceed by a factor of 1.8 in the downwind, 1.4 in the crosswind and 1.25 in the upwind direction; at $0.5 \mathrm{AU}$ these ratios attain even higher values of $2.4,1.7$ and 1.4, respectively. Still closer to the Sun, at $0.01 \mathrm{AU}$, the departures may reach an order of magnitude upwind and crosswind, and two orders of magnitude in the cone, but one must keep in mind that inside $1 \mathrm{AU}$ a significant role in the helium destruction is played by electron impact ionization, which was excluded from this analysis, but which may change significantly the density pattern in the immediate solar neighbourhood. There is very little data available on the long time-scale evolution of the electron impact ionization rate during the solar cycle. If, however, this rate is not significantly variable (as measurements performed at $1 \mathrm{AU}$ in ecliptic by ACE seem to suggest, McMullin et al., in preparation), then the amplitude of helium density inside $\simeq 1 \mathrm{AU}$ should be smaller than presented here because the amplitude $B$ of the net ionization rate $\beta_{\text {net }}(t)=\beta_{\mathrm{EUV}}(t)+\beta_{\mathrm{el}-\text { imp }}$ will be smaller than the amplitude $B_{\mathrm{EUV}}$ of the photoionization rate $\beta_{\mathrm{EUV}}(t)$ :

$$
\begin{aligned}
B_{\mathrm{EUV}} & =\max \left|\frac{\beta_{\mathrm{EUV}}(t)-\left\langle\beta_{\mathrm{EUV}}\right\rangle}{\left\langle\beta_{\mathrm{EUV}}\right\rangle}\right| \\
& >\max \left|\frac{\beta_{\mathrm{EUV}}(t)+\beta_{\mathrm{el}-\mathrm{imp}}-\left\langle\beta_{\mathrm{EUV}}+\beta_{\mathrm{el}-\mathrm{imp}}\right\rangle}{\left\langle\beta_{\mathrm{EUV}}+\beta_{\mathrm{el}-\mathrm{imp}}\right\rangle}\right| \\
& =\max \left|\frac{\beta_{\mathrm{EUV}}(t)-\left\langle\beta_{\mathrm{EUV}}\right\rangle}{\left\langle\beta_{\mathrm{EUV}}\right\rangle+\beta_{\mathrm{el}-\mathrm{imp}}}\right|=B .
\end{aligned}
$$

We stress that we mean the long time-scale variations of the electron ionization rate, and not the local daily fluctuations, which inside $1 \mathrm{AU}$ may reach an order of magnitude.

A comparison of Figs. 4 and 5 shows that the density amplitude increases slowly but visibly from the upwind direction with the increase in the offset angle $\theta$ even outside the helium cone. However, at the distances where the GAS/ Ulysses instrument is operating (mainly $3-5 \mathrm{AU}$ almost at crosswind) the departures of helium density from the mean 


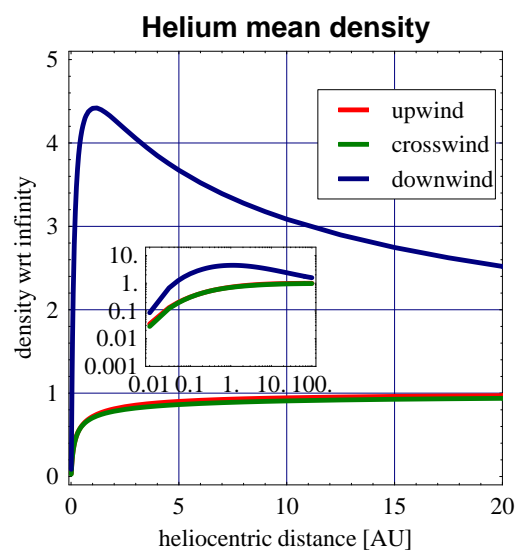

Helium density variations upwind

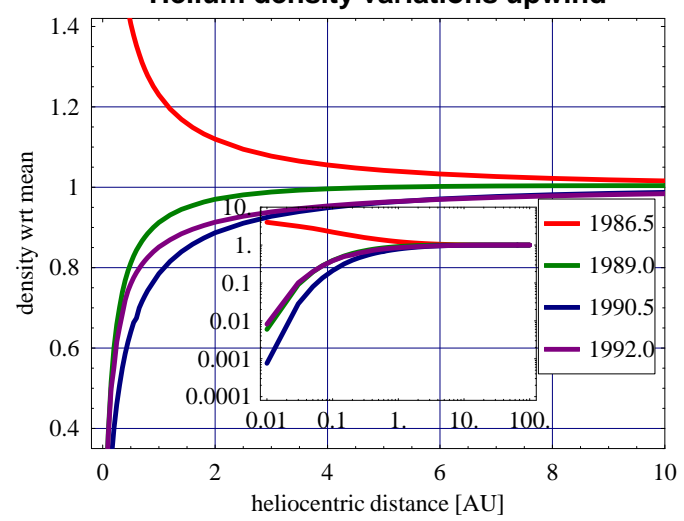

Helium density variations downwind

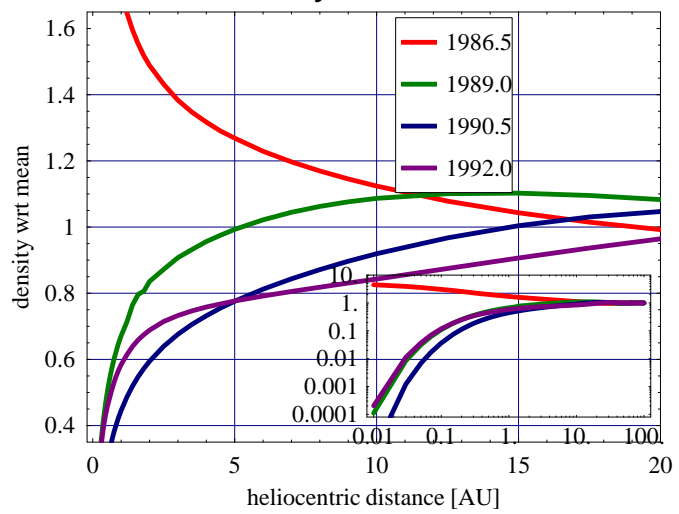

Helium density variations crosswind

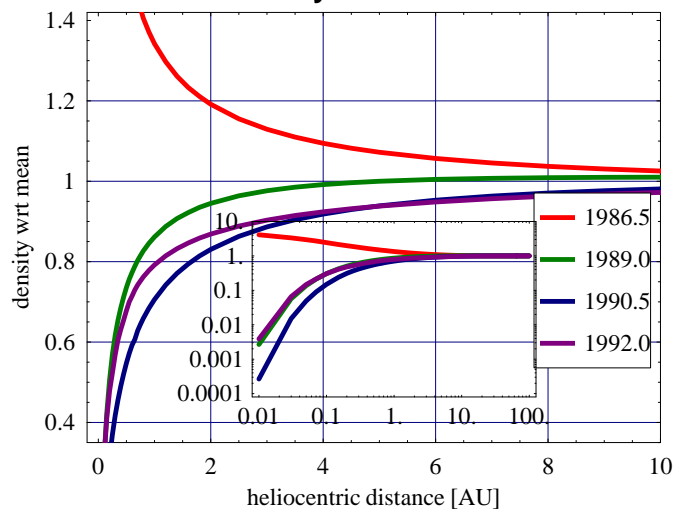

Fig. 3. Evolution of helium density during the solar cycle. Upper left panel: mean density along the upwind, crosswind and downwind directions, shown as a fraction of the density at infinity. The other three panels: relative departures of the density from the mean value presented in the upper left panel, shown along the upwind (lower left panel), crosswind (lower right panel) and downwind (upper right panel) directions close to the Sun for selected epochs during the solar cycle. The epochs are indicated in the boxes inside the panels. Insets show the same qunatities but presented in the log-log scale for the whole range of helicoentric distances from 0.01 to 100 AU from the Sun.

are quite small. This is the explanation of the fact that GAS/ Ulysses has not observed any bold time dependent effects in helium density (Witte et al., 1996, 2003) although a confirmed and significant variation of the photoionization rate has been detected by direct Sun observations (Ogawa et al., 1997, 1998; Judge et al., 1998, McMullin et al., in preparation).

The departures of helium density from the mean value are roughly anticorrelated with the changes in the ionization rate (high ionization rate implies low helium density and vice versa) and this anticorrelation is essentially manifest in the whole upwind hemisphere. However, the anticorrelation is not perfect since the gas needs some time to adjust to the varying ionization rate. The delay depends mainly on the linear distance from the Sun and, surprisingly, very little of the offset angle from the upwind direction. The phase shift problem is illustrated in Fig. 7. The upper left panel of this figure presents the calculation grid: densities were evaluated with $0.5 y$ resolution along the gray lines parallel to the flow axis at the distances $\rho=0.5,1,3,5$, and $10 \mathrm{AU}$. The points discussed below are at the intersections of these lines with radial lines inclined at angles $\sim 25^{\circ}$ and $\sim 155^{\circ}$ (marked with asterisks), as well as at the corresponding distances crosswind.

Close to the Sun the correlation is almost perfect and there is very little phase shift between density and the ionization rate, regardless of the offset angle from the upwind direction - see the middle panel in the left column in Fig. 7, where the situation at $1 \mathrm{AU}$ is shown. When the distance from the Sun increases, however (see the panel just below the former one), more visible phase shifts appear. At about $5 \mathrm{AU}$ from the Sun, they are almost independent on the offset angle. A more systematic presentation of the dependence of the phase shift on the heliocentric distance is provided in the right-hand column of Fig. 7. The three panels illustrate the phase shift expected at 25, 90 and 155 degrees from upwind at heliocentric distances from $1 \mathrm{AU}$ to $10 \mathrm{AU}$. Close to the Sun, the phase shifts are very similar to each other regardless of the offset angle. The shifts increase with the increase of the heliocentric distance (compare the violet and pink curves between the panels in this column) but still very little differentiation of the phase shift with the offset angle can be seen. 


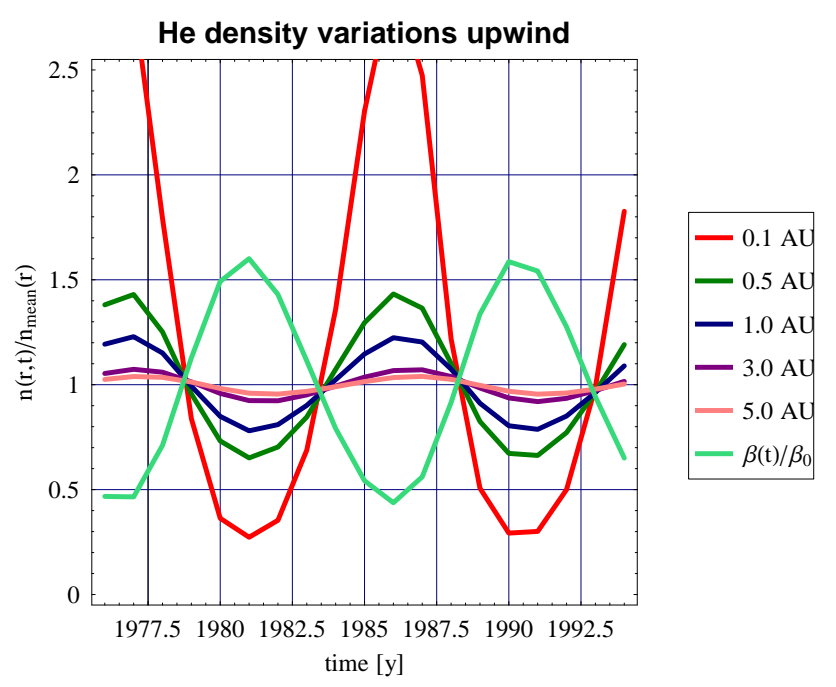

Fig. 4. Deviations of helium density from the mean values during the solar cycle, at the upwind axis at selected distances from the Sun: shown is the quantity $n\left(r, \theta=0^{\circ}, t\right) / n_{\text {mean }}\left(r, \theta=0^{\circ}\right)$ computed at the distances indicated in the box next to the figure. Also shown (for reference purposes) is the variation of the photoionization rate assumed to the mean value, marked " $\beta(t) / \beta_{0}$ ".

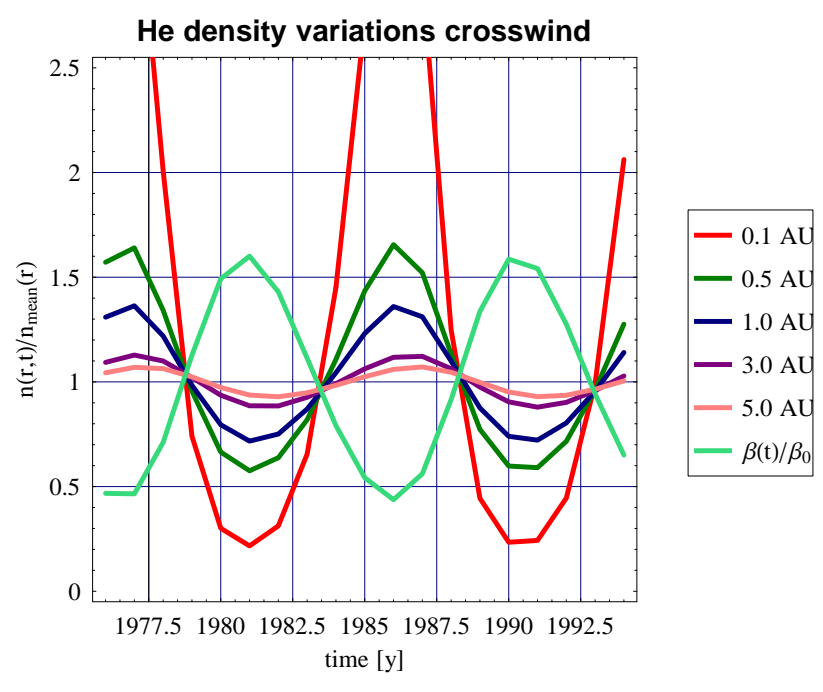

Fig. 5. Deviations of helium density from the mean at the crosswind axis; see caption to Fig. 4.

Another important effect of the variable ionization rate is reflected in the variations of the helium cone relative height, shown in Fig. 8. In the figure, the quantities $h_{\text {mean }}(r)=$ $n_{\text {mean }}\left(r, 180^{\circ}\right) / n_{\text {mean }}\left(r, 0^{\circ}\right)$ for the mean model and $h(r, t)=n\left(r, 180^{\circ}, t\right) / n\left(r, 0^{\circ}, t\right)$ for four selected epochs during the solar cycle are presented. The helium cone height is important for studies of interplanetary helium backscatter resonance glow and, in particular, for derivation of helium density and temperature values. The maximum of the cone height for the mean density occurs typically at about $1 \mathrm{AU}$ from the Sun but can vary during the solar cycle from about

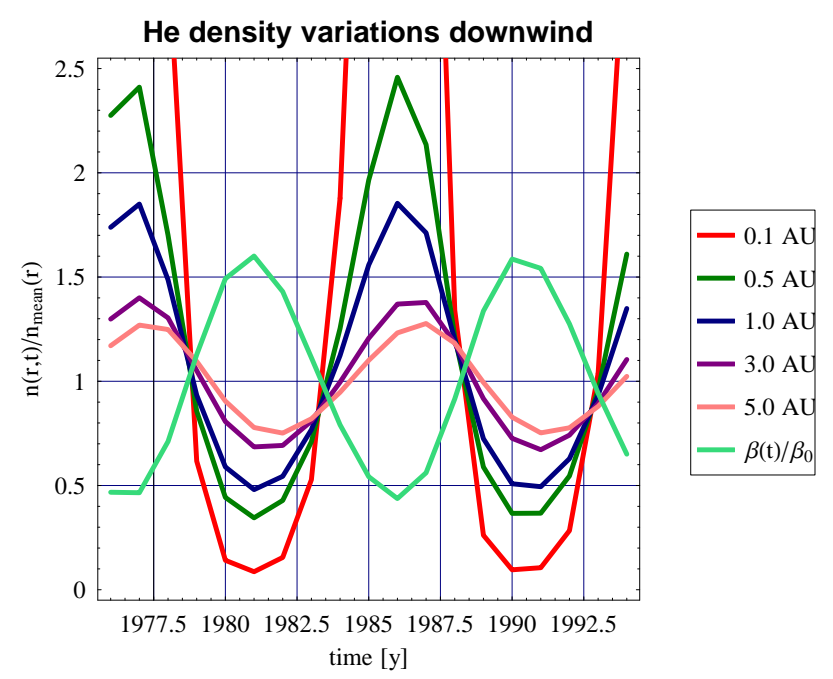

Fig. 6. Deviations of helium density from the mean at the downwind axis (i.e. in the cone), see caption to Fig. 4.

0.25 AU to about 2 AU. Even more important are quite significant variations of the magnitude of the relative cone height $h(r, t) / h_{\text {mean }}(r)$ in the region relevant for optical studies, as shown in Fig. 9. They are at the level of about 1.5 at $1 \mathrm{AU}$ and further away from the Sun they decrease more or less linearly with the heliocentric distance, reaching about $10 \%$ at $15 \mathrm{AU}$.

We conclude this section by discussing the possible influence of small-scale fluctuations of the helium ionization rate owing to, for example, variations of helium charge exchange rate with solar wind particles. As indicated by Ruciński et al. (1996), charge exchange ionization makes up only a very small percentage of the net ionization rate, at least in the slow solar wind. We checked whether a small modulation of the photoionization rate adopted in this study will be seen at all in the resulting helium density.

To that end, we define a modulated helium ionization rate as:

$\beta_{m}(t)=\left(1+0.03 \cos \omega_{m} t\right) \beta(t)$,

where $\beta(t)$ is the function presented in Fig. 1 and $\omega_{m}=$ $2 \pi / 1$ year, and we calculate helium density upwind, crosswind and downwind every month for time intervals of about 2 years long during solar minimum and maximum epochs. For comparison, we perform analogous calculations with the ionization rate described by the function $\beta(t)$.

The results of this test are collected in Fig. 10. The upper row of panels presents the appropriate intervals of the two ionization rates for the minimum (left) and maximum (right) of the solar cycle and the middle and lower rows show deviations of the density calculated with the use of the modulated ionization rate $\beta_{m}(t)$ with respect to the densities calculated with the use of $\beta(t)$. The left-hand column corresponds to solar minimum and the right-hand column to solar maximum conditions. The middle row is for the heliocentric distance $0.5 \mathrm{AU}$ and the lower row for $1 \mathrm{AU}$; the quantity shown 

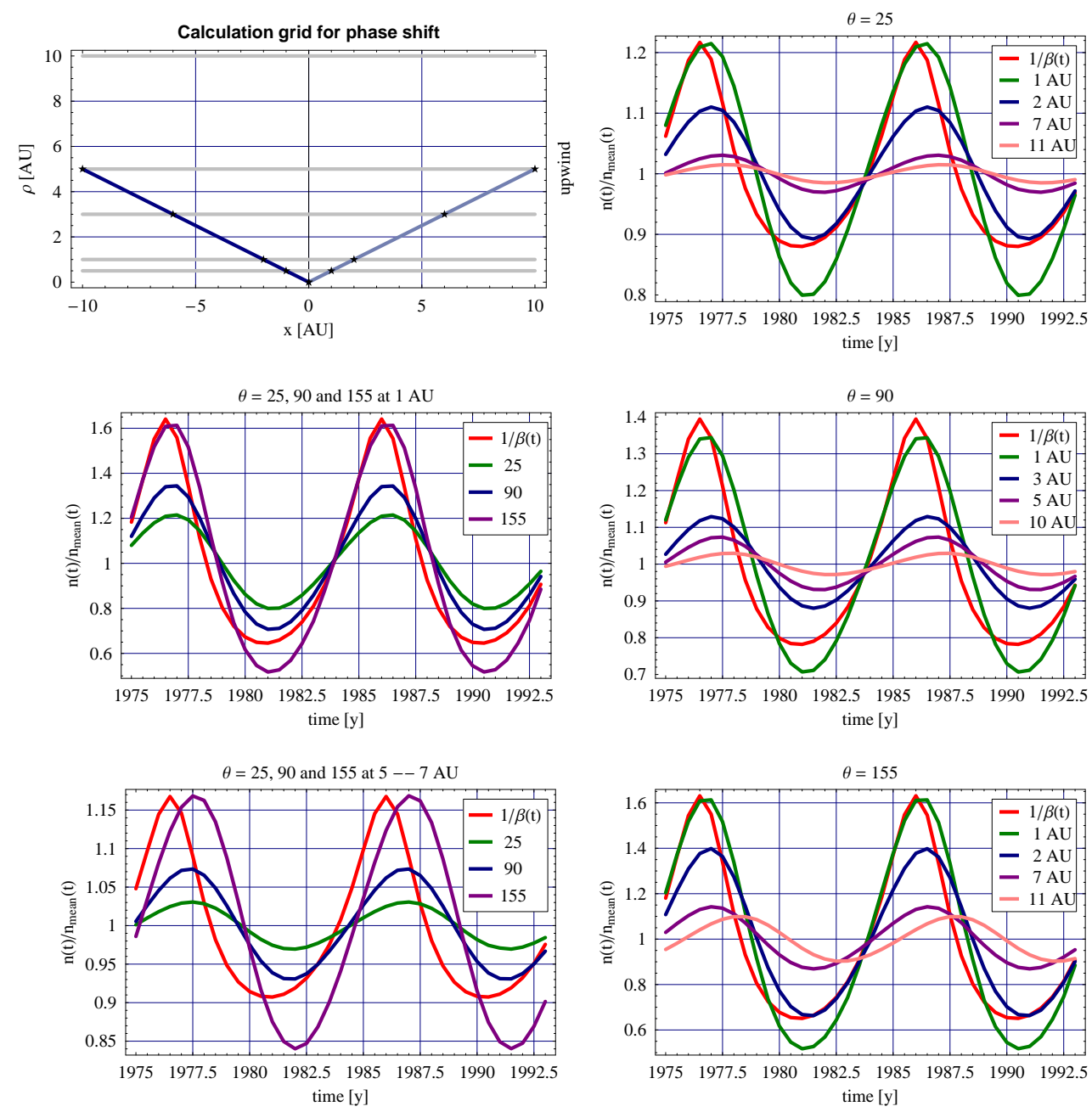

Fig. 7. Dependence of phase shift between time variations of the helium ionization rate and resulting deviations of helium density from the mean value. Upper left panel: calculations grid. The horizontal axis is the upwind - downwind direction, upwind is to the right. The vertical axis is the $\rho$ coordinate in the cyllindric reference system. The intersection of the horizontal gray lines with the blue slant lines, marked with asterisks, are places where calculations were made marked as $\theta=25^{\circ}$ and $\theta=155^{\circ}$. Additionally, the calculations were performed for the intersection of the crosswind direction $\theta=90^{\circ}$ with the gray lines. In the remaining panels: the red line is appropriately rescaled lifetime against ionization $T(t) / T_{\text {mean }}$ scaled to the mean time against ionization $(T(t)=1 / \beta(t))$, presented here for reference of the phase of the ionization rate. In the left-hand column, middle and lower panels: phase shifts of the deviations of helium density from the solar-cycle mean are shown for the 25, 90 and $155^{\circ}$ off apex at $1 \mathrm{AU}$ (middle) and $5 \mathrm{AU}$ from the Sun (lower). Right hand column: comparison of the phase shifts at various heliocentric distances at 25 (upper), 90 (middle) and $155^{\circ}$ (lower panel) from the upwind direction at various heliocentric distances: the effect of the increase in the phase shift with the heliocentric distance and its negligible dependence on the offset angle is presented.

is $n_{\text {modulated }}(r, \theta, t) / n_{\text {non-modulated }}(r, \theta, t)$ for the upwind, crosswind and downwind axes.

The results show that when the amplitude of fluctuations scales linearly with the magnitude of the ionization rate, their effect is stronger during solar maximum than during minimum. However, they appear to modify noticeably the helium density only inside $1 \mathrm{AU}$ in the cone and inside $0.5 \mathrm{AU}$ elsewhere; the amplitude of fluctuations is just a few percent and in the cone it is approximately two-fold higher than elsewhere. Since, however, this is the region where the electron impact ionization must be taken into account as it constitutes a large, if not dominant component of the net ionization rate, we conclude that fluctuations of this kind can safely be ignored except in the regions closest to the Sun, where even small changes in the ionization rate can induce noticeable changes in the density values.

\subsection{Evolution of $\mathrm{He}^{+}$pickup ions during solar cycle}

The $\mathrm{He}^{+}$pickup ion flux in the upwind hemisphere attains its maximum at $\simeq 0.1 \mathrm{AU}$ from the Sun and decreases beyond, while in the cone region it starts decreasing somewhat farther, beyond $\simeq 0.3 \mathrm{AU}$ (see Fig. 11, where the radial behavior of the average flux during the solar cycle in the up- 


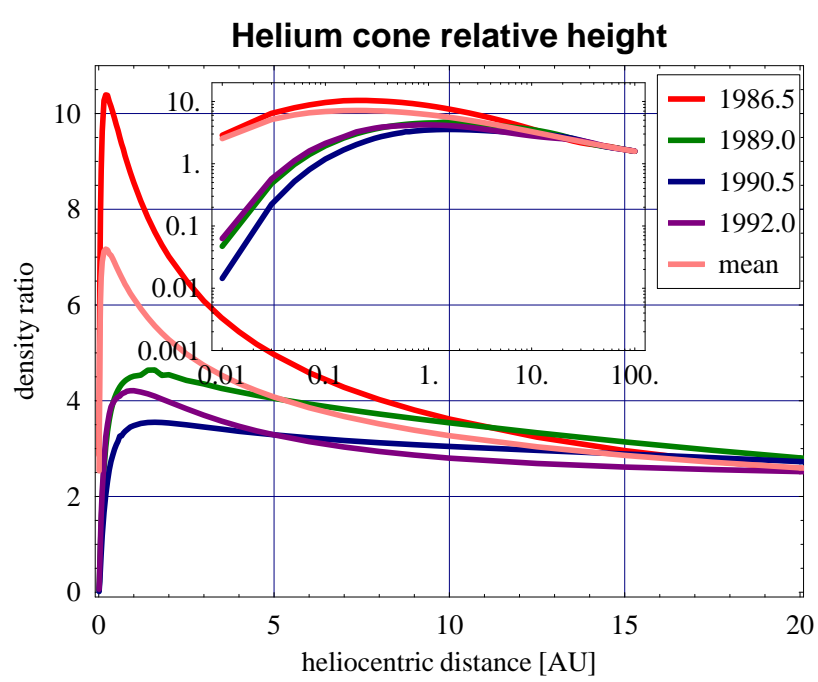

Fig. 8. Relative height of helium cone, defined as downwind to upwind density ratio, for four selected epochs during the solar cycle. The epochs are marked in the box inside the figure. Inset: same quantity in log-log scale, full range of heliocentric densities.

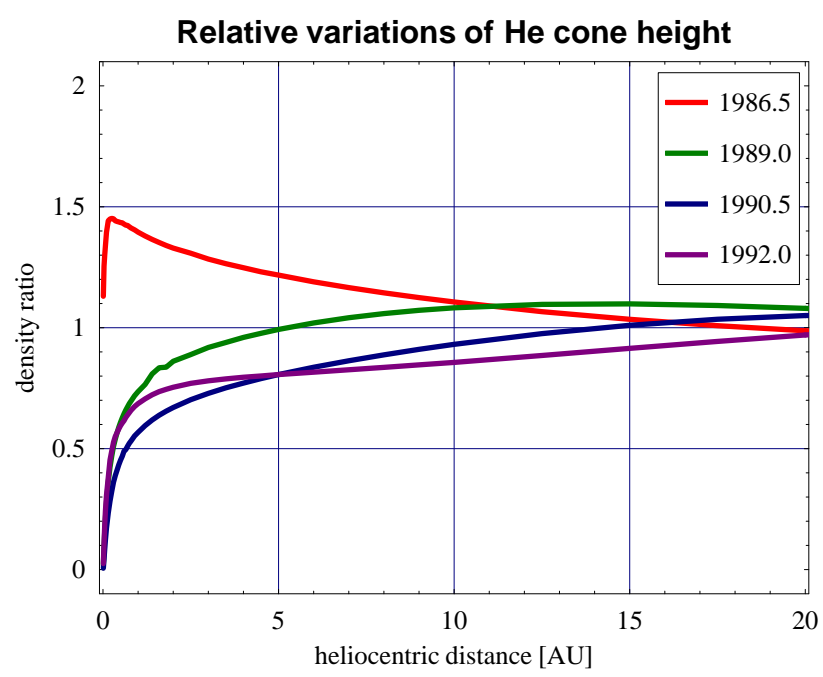

Fig. 9. Variations of the relative helium cone height during the solar cycle with respect to the mean helium cone height as a function of the heliocentric distance for selected epochs.

wind, crosswind and downwind directions is presented). The average flux differs from the MSM flux but the radial behavior of this discrepancy is not similar to the corresponding differences in density (see Fig. 12). Except in the cone, the discrepancies stay within $20 \%$, and outside $0.2 \mathrm{AU}$ even within $10 \%$. Beyond $10 \mathrm{AU}$ they basically disappear. In the cone, however, they reach a factor of 2 at $0.1 \mathrm{AU}$ and come down below $10 \%$ beyond $0.4 \mathrm{AU}$, and farther away are generally larger than outside the cone up to the distance of about $50 \mathrm{AU}$.

Close to the Sun, the deviations of the flux from the mean are anticorrelated with the variations of the ionization rate, while far away from the Sun the correlation is positive. It can be seen in Figs. 13 and 14, where the evolution of the $\mathrm{He}^{+}$ PUI flux at selected distances from the Sun, normalized to the flux from the stationary model, is presented correspondingly for the crosswind and downwind directions. The variations upwind are very similar to the variations crosswind and thus are not shown.

The transition region between the two types of correlation occurs at 2-5 AU in the cone but as close as $<1$ AU elsewhere. Ulysses is operating in the region of positive correlation, i.e. the variations of the $\mathrm{He}^{+}$PUI flux should follow the variations of the photoionization rate and the expected amplitude is of the order of $40 \%$ during the solar cycle, depending somewhat on the distance from the Sun.

The reversal of correlation is well illustrated in Figs. 15 and 16 , where the same quantity

$Q_{F}(r, \theta, t)=F(r, \theta, t) / F_{\text {mean }}(r, \theta)$

is presented for selected epochs during the solar cycle for $\theta=90^{\circ}$ and $\theta=180^{\circ}$ (crosswind and downwind, respectively) as a function of heliocentric distance. $F(r, \theta, t)$ is the magnitude of the pickup flux at the heliocentric distance $r$ and offset angle $\theta$ at time $t$, and $F_{\text {mean }}(r, \theta)$ is the pickup flux calculated using the classical hot model with the ionization rate equal to the mean over the solar cycle. Inside $\sim 1 \mathrm{AU}$ and outside $\sim 5 \mathrm{AU}$ in the cone and, correspondingly, $0.3 \mathrm{AU}$ and $3 \mathrm{AU}$ elsewhere variations of $Q_{F}(r, \theta, t)$ exceed $50 \%$ but in the region penetrated by Ulysses and Earth-bound spacecraft they stay at the mild level of $\sim 50 \%$. One must keep in mind, however, that superimposed on the long-scale variations related to global solar cycle variations of the photoionization rate there should appear instantaneous variations of the pickup flux owing to instantaneous variations of the local production rate $\beta_{\text {prod. }}$. This holds as well for Ulysses as for any other mission, everywhere in the heliosphere.

\subsection{Solar cycle changes in the spectral characteristics of he- lium pickup ion distributions}

In the previous sections of this paper we have shown how the solar cycle-induced variation of the helium photoionization rate influences the radial density profiles of interplanetary atomic helium in the upwind, crosswind and downwind regions of the heliosphere. We demonstrated how much variable over the solar activity cycle these density profiles are. This, however, means that resulting local $\mathrm{He}^{+}$pickup ion injection rates are also subject to strong variations. We are going to study the consequences of this fact in the coming paragraphs.

It should be mentioned here that studies of the PUI reactions on a delta-type increase in the solar photoionization rate have already been published earlier by Isenberg and Lee (1995). Following a sudden increase in the photoionization rate, these authors predict a $\mathrm{He}^{+}$PUI density enhancement lasting for times of the order of the duration of the increased ionization plus the convection time of the solar wind to the position $r$. This increase is then followed by a PUI density decrease with a recovery period of the order of the travel time 

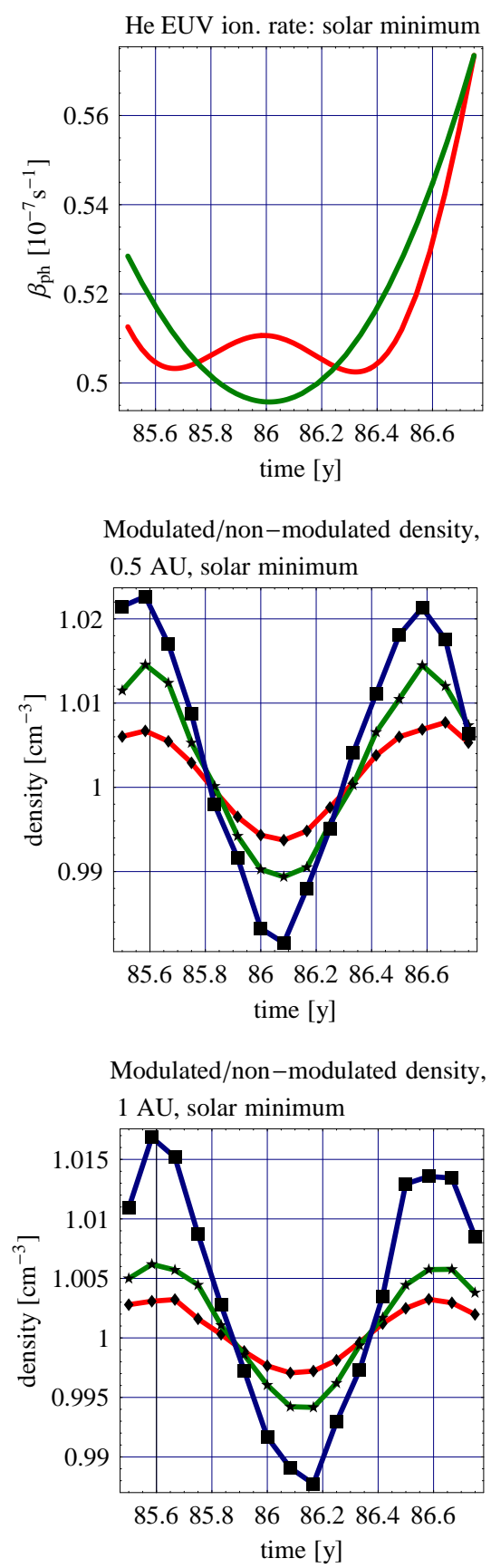

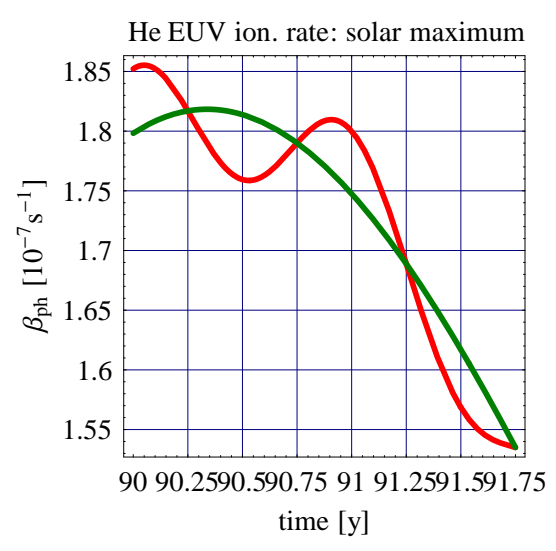

Modulated/non-modulated density, $0.5 \mathrm{AU}$, solar maximum

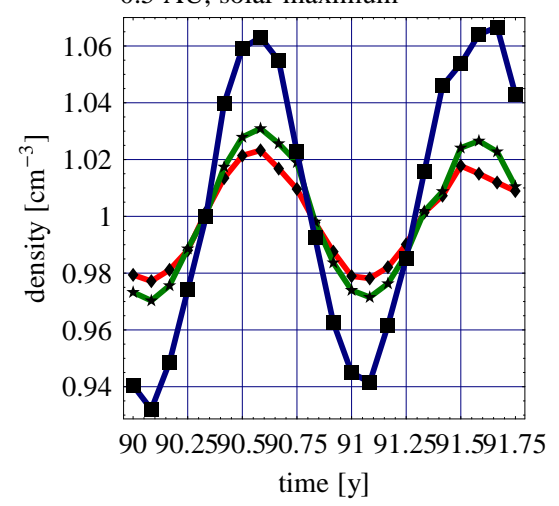

Modulated/non-modulated density, $1 \mathrm{AU}$, solar maximum

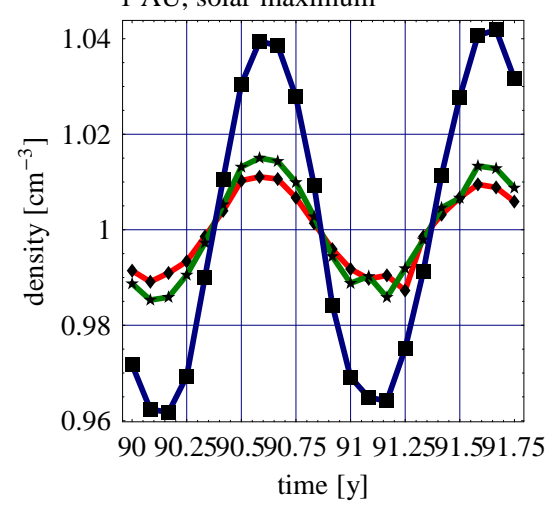

Fig. 10. Effects of small periodic fluctuations of ionization rate on helium density during solar minimum and maximum. Upper row: the modulated (red) and non-modulated ionization rates; the modulated rate is given by Eq. (10) and the non-modulated rate is presented in Fig. 1. Middle and lower rows: ratios of modulated to non-modulated densities at $0.5 \mathrm{AU}$ from the Sun (middle row) and at $1.0 \mathrm{AU}$ from the Sun (lower row). The red, green and blue lines in the two lower rows correspond to the upwind, crosswind, and downwind directions. The left-hand column is for solar minimum conditions and the right-hand column to the solar maximum conditions; times are indicated at the horizontal axes.

of neutral helium atoms over this distance $r$. These earlier results, however, cannot be directly compared with the results obtained here, because in our study the typical period of solar activity change is long compared to solar wind convection and neutral helium travel time periods (see Eq. 7), in contrast with the assumption adopted by Isenberg and Lee (1995).
Since observational access to PUI properties is usually given by measurements of the PUI distribution function $f_{\text {pui }}$ rather than of the total PUI flux, which only is derived as a secondary quantity, it is very interesting to look at specific spectral features of model $\mathrm{He}^{+}$PUI distribution functions and their variation over the solar cycle. Following the ap- 
Helium PUI mean flux

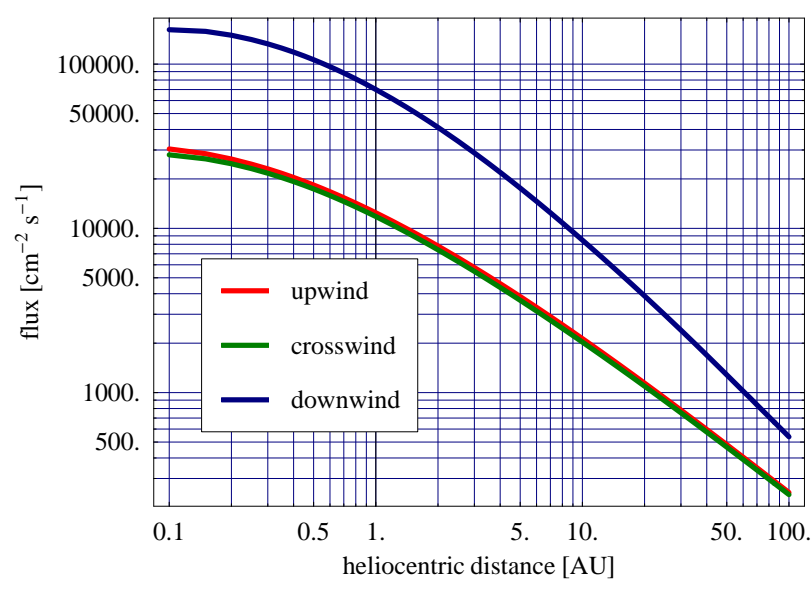

Fig. 11. Mean flux of $\mathrm{He}^{+}$pickup ions upwind, crosswind and downwind in the log-log scale for the He density at infinity equal to 1 atom $\mathrm{cm}^{-3}$ and stationary ionization rate equal to the mean value of the function presented in Fig. 1.

\section{Mean PUI flux/PUI flux for mean ionization}

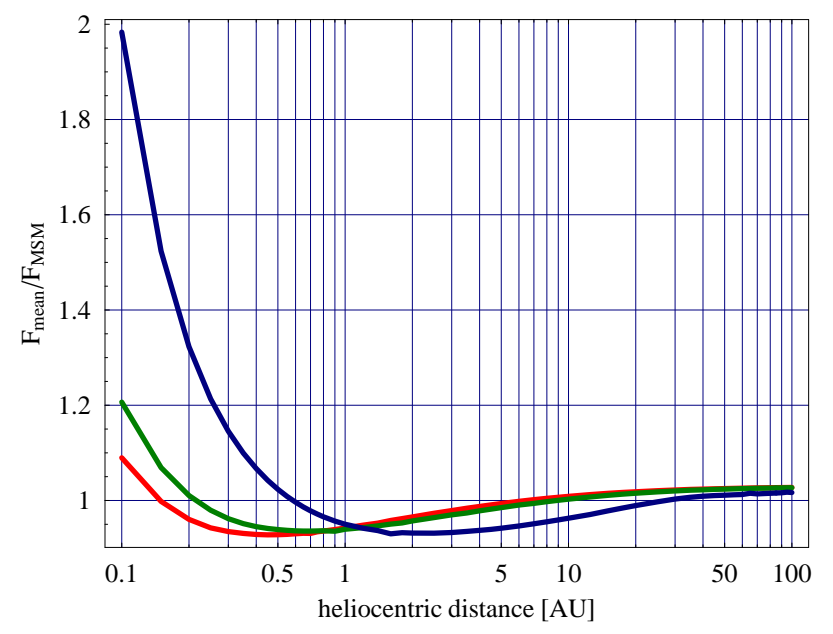

Fig. 12. Ratios of the He PUI flux averaged over solar cycle to the stationary flux calculated for the ionization rate averaged over the solar cycle for the upwind (red), crosswind (green), and downwind (blue) directions.

proach by Vasyliunas and Siscoe (1976), where pickup ions after injection are assumed to become immediately pitchangle isotropized and then convected with the solar wind, simply undergoing adiabatic deceleration during its expansion, we have the following expression for the distribution function:

$f_{\text {pui }}(r, \theta, w)=\frac{3}{8 \pi V^{4}} \frac{\beta_{\mathrm{pi}} r_{\mathrm{E}}^{2} w^{-3 / 2}}{r} n_{\mathrm{He}}\left(r w^{3 / 2}, \theta\right)$.

Here, $\beta_{\mathrm{pi}}$ is the actual reference value of the photoionization rate at $r=r_{\mathrm{E}}, V$ is the solar wind velocity, and $w=$

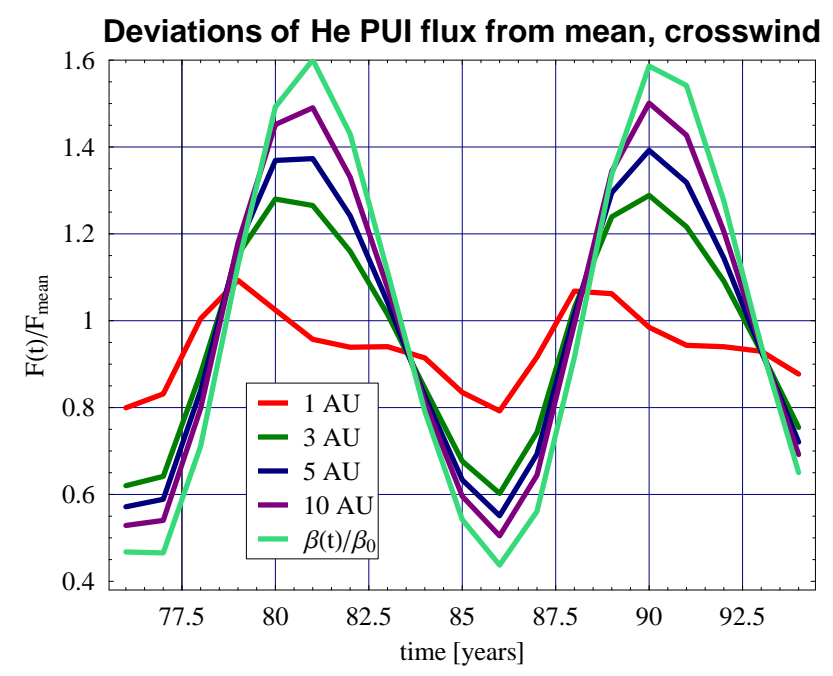

Fig. 13. Deviations of $\mathrm{He}^{+}$pickup flux from the solar-cycle mean value, for the crosswind axis at selected heliocentric distances, indicated in the inset box. For reference, deviations of the photoionization rate from the mean value $\beta(t) / \beta_{\text {mean }}$ are shown, as indicated in the box.

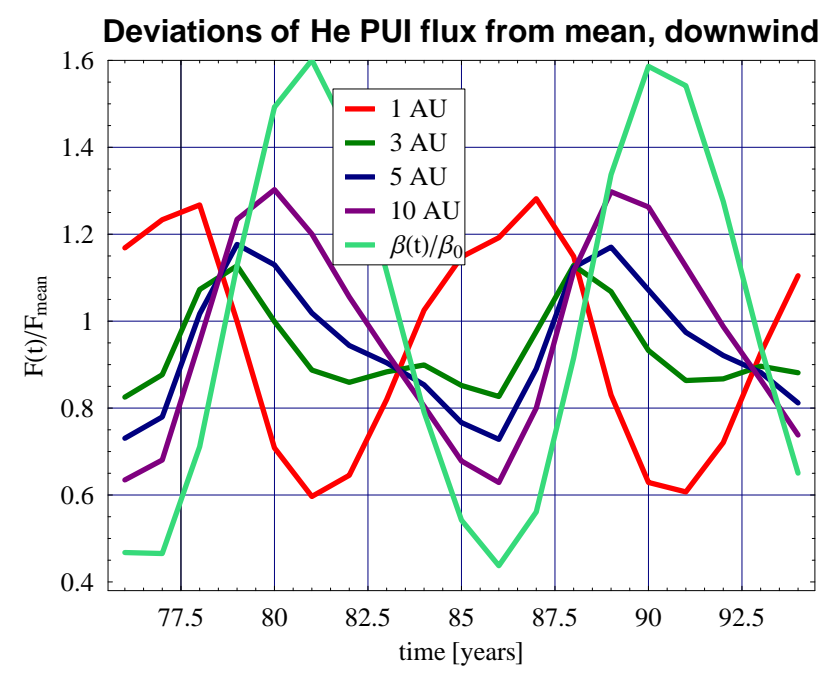

Fig. 14. Deviations of $\mathrm{He}^{+}$pickup flux from the solar-cycle mean for the downwind axis at several heliocentric distances, indicated in the inset box. For reference, deviations of the ionization rate from the mean value $\beta(t) / \beta_{\text {mean }}$ are shown, as indicated in the box.

$v / V \leq 1$ is the PUI velocity magnitude in the solar wind rest frame, normalized by $V$. The quantity $n_{\mathrm{He}}\left(r w^{3 / 2}, \theta\right)$ is the actual helium atom density at a reduced distance $r^{*}=r w^{3 / 2}$ taken in the direction $\theta$, where the PUIs having velocity $w$ at distance $r$ are injected. The above given solution for the function $f_{\text {pui }}(r, \theta, w)$ is a reasonable approach in regions far enough from CIRs and at small solar distances from the Sun, where no energy diffusion of pickup ions due to nonlinear wave-particle interactions needs to be considered. Close to CIRs and at larger distances this assumption is clearly violated, as proven by many suprathermal spectral wings oc- 


\section{Deviations of He PUI flux from mean, crosswind}

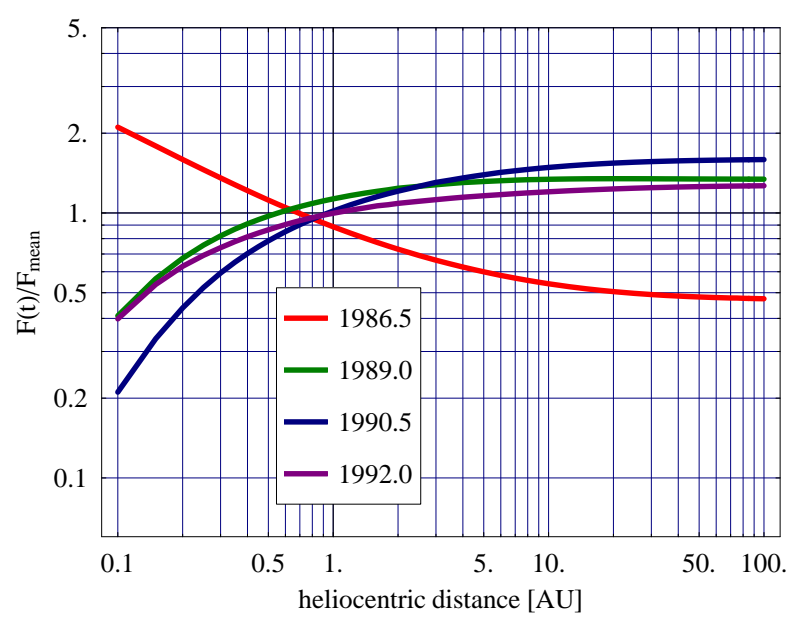

Fig. 15. The actual flux of $\mathrm{He}^{+}$pickup ions with respect to the flux averaged over time, for selected epochs during the solar cycle, presented as function of heliocentric distance along the crosswind axis in the log-log scale. The epochs are color-coded in the inset box.

\section{Deviations of He PUI flux from mean, downwind}

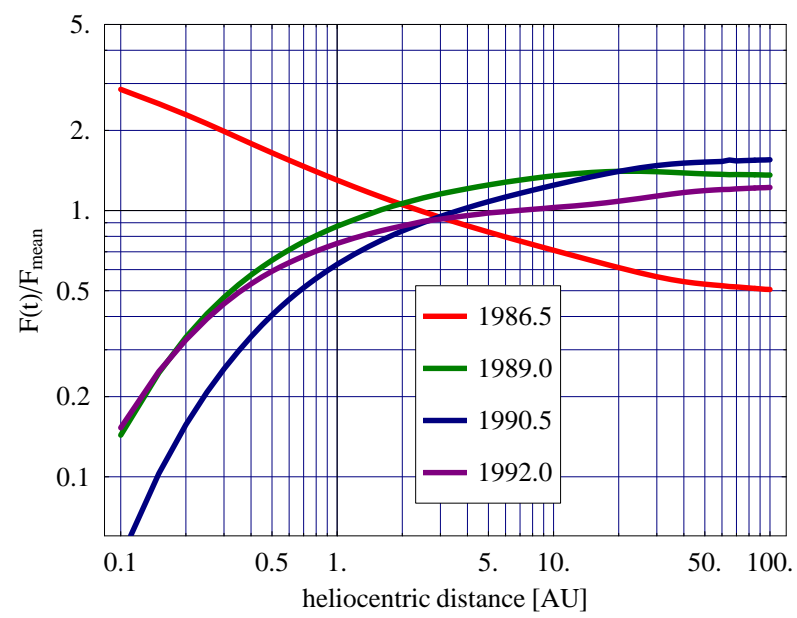

Fig. 16. The actual flux of $\mathrm{He}^{+}$pickup ions with respect to the flux averaged over time, for selected epochs during the solar cycle, presented as a function of heliocentric distance along the downwind axis in the log-log scale. The epochs are color-coded in the inset box.

curring in observed $\mathrm{He}^{+}$pickup ion spectra presented by Gloeckler (1999, 2003).

Staying, however, within the limits of the above mentioned approximation, it is evident from Eq. (11) that the resulting slope of the distribution function $\partial f_{\text {pui }} / \partial w$ directly contains information on the helium density gradient at the associated injection place, since the following relation is valid:

$\frac{\partial f_{\text {pui }}}{\partial w}=\frac{3}{2} f_{\text {pui }}\left(w^{1 / 2} r\left|\frac{\partial \ln }{\partial r} n_{\mathrm{He}}\right|_{r w^{3 / 2}}-\frac{1}{w}\right)$.

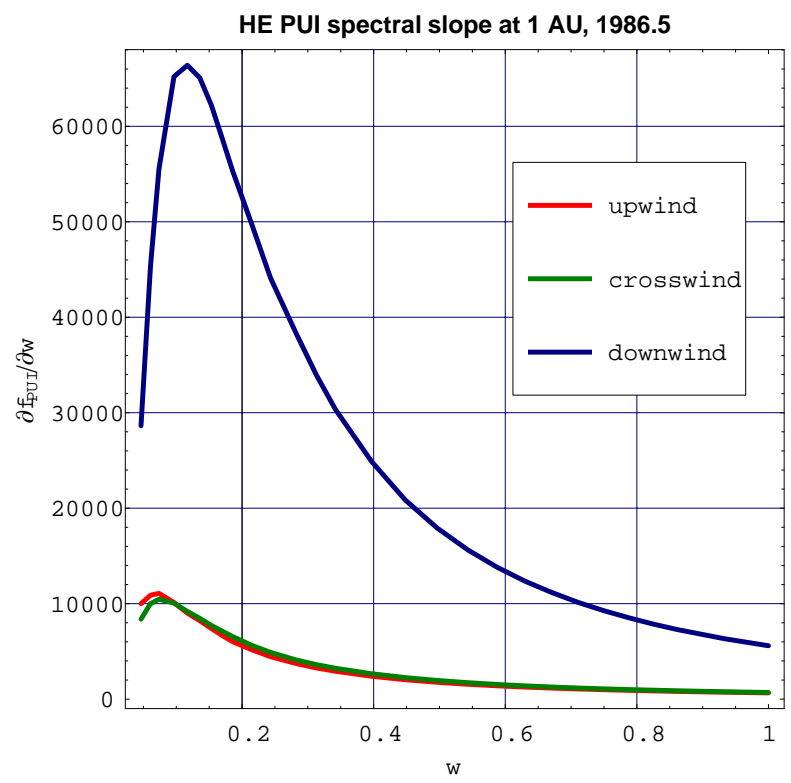

Fig. 17. Slope of $f_{P U I}(r, \theta, t)$ at $r=1 \mathrm{AU}$, defined in Eq. (12), as a function of speed normalized to solar wind speed: $w=v / V$ in the directions upwind, crosswind, and downwind, color-coded in the inset box, for the epoch of solar minimum $(t=1986.5)$.

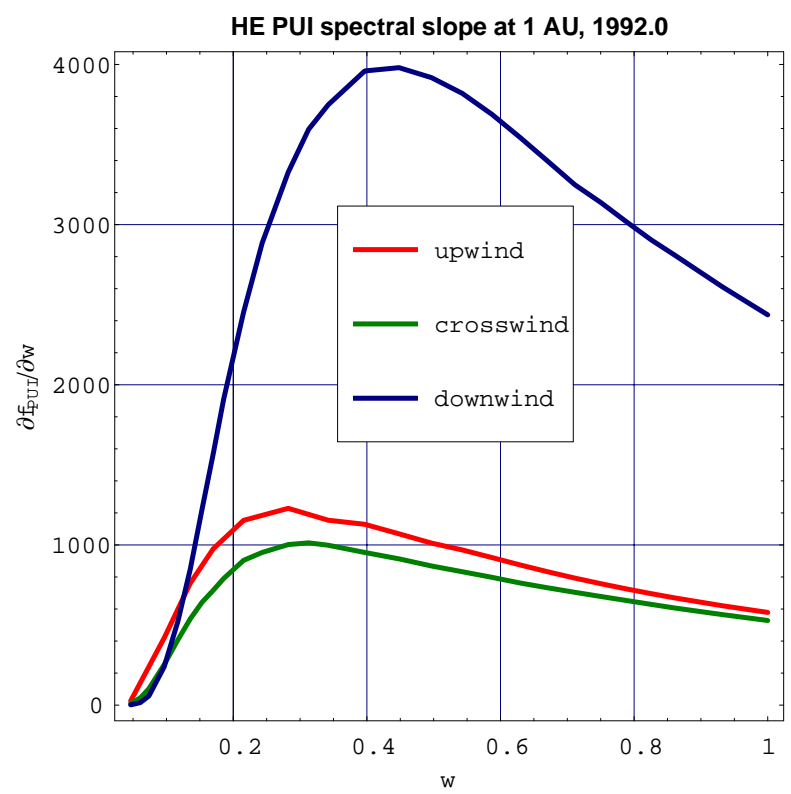

Fig. 18. As Fig. 17 but for solar maximum epoch (1992.0).

Since both helium density and its gradient are functions of $\theta$ and of the actual time $t$ within the solar cycle, the slope of the distribution function as derived in Eq. (12) is reflected in the actual appropriate spectral observation changes, both with $\theta$ and with the time during the solar cycle.

The actual helium density profile (slope) leading to a specific PUI distribution function can be reconstructed by a kind of inversion technique from the following expression for 


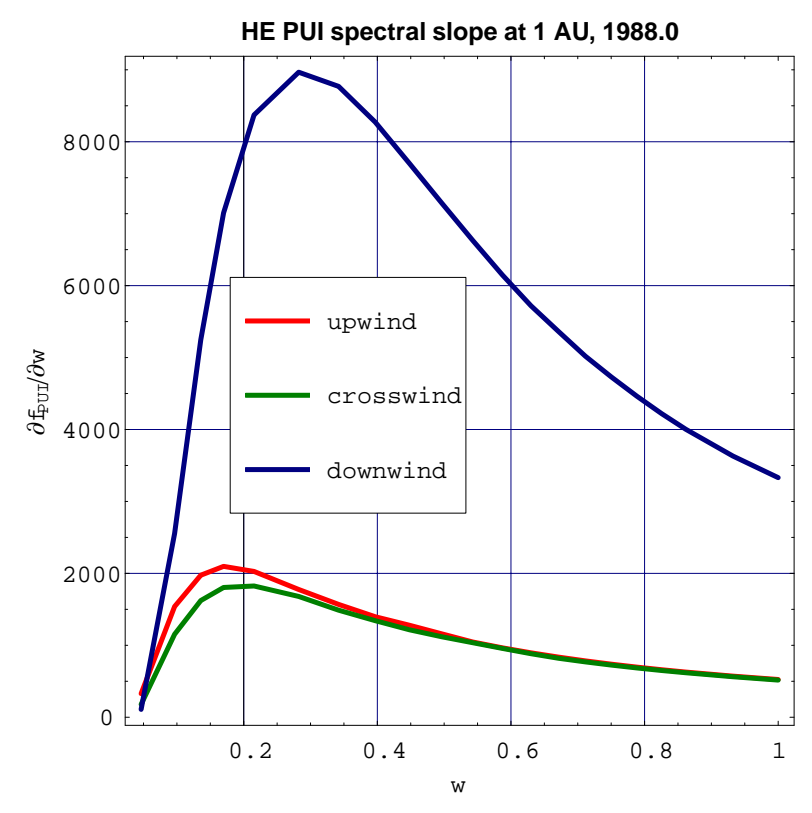

Fig. 19. As Figs. 17 and 18 but for an intermediate phase of the solar cycle $(t=1988.0)$.

$\partial n_{\mathrm{He}} / \partial r$, derived from the slope of the function $f_{\text {pui }}$ :

$\left|\frac{\partial \ln }{\partial r} n_{\mathrm{He}}\right|_{r w^{3 / 2}}=\frac{2}{3} \frac{1}{r \sqrt{w}}\left(\frac{\partial}{\partial w} \ln f_{\text {pui }}+\frac{3}{2} \frac{1}{w}\right)$.

The actual form of the slope of distribution function $f_{\text {pui }}(r, \theta, t)$ evaluated on the basis of Eq. (13) and the theoretical helium densities $n_{\mathrm{He}}(r, \theta, t)$ discussed in the previous subsection is shown in the forthcoming figures. In particular, the change in the slope as a function of time $t$ during the course of the solar cycle can explicitly be studied in the results displayed in Figs. 17 through 20.

In Fig. 17 one can see the slope profiles of $f_{\text {pui }}(r, \theta, w)$ at $r=r_{\mathrm{E}}$ as a function of $w$ in the upwind, crosswind, and downwind direction. The peak of the quantity presented in the figure corresponds to the highest slope of the distribution function $f_{\text {pui }}(r, \theta, w)$ at $r=r_{\mathrm{E}}$. The time for this evaluation hereby is 1986.5 (i.e. solar minimum). As one can clearly see in this figure, both the slope and the absolute spectral intensity of $f_{\text {pui }}$ (which is the field under the profile) strongly differ from upwind to downwind positions. In Fig. 18 we have shown analogous quantities, but now evaluated for the time 1992.0 (i.e. just after solar maximum in the phase of decreasing activity). Again, there are strong differences with $\theta$ both in spectral intensities and slopes, but more important is that in comparison with Fig. 17 the maximum slope of the distribution function in all cases appears shifted towards larger values of $w$. Figure 19 shows analogous data as Figs. 17 and 18 but this time for the time 1988.0 (i.e. the time of increasing solar activity). The curves are qualitatively similar to those shown in Fig. 19 but all spectral slopes in this case are higher and the spectral peaks have moved to smaller values of $w_{\max }$.

Figures 20 and 21 display the slopes to be expected at four different phases of the solar activity cycle at $1 \mathrm{AU}$ in

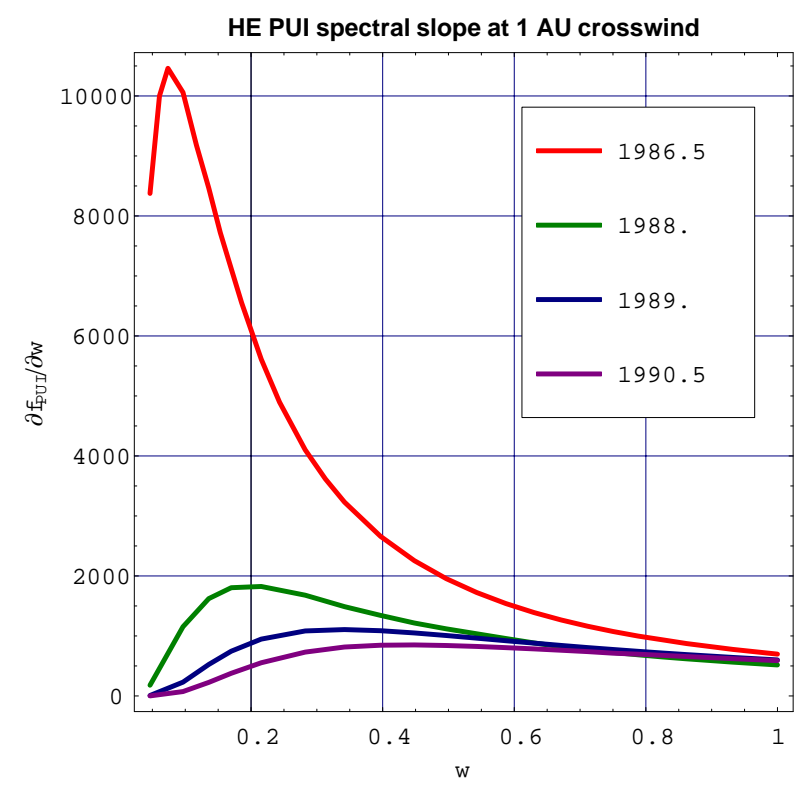

Fig. 20. Slopes of $f_{\text {PUI }}$ at four different phases of the solar cycle at $1 \mathrm{AU}$, crosswind position; $w=v / V$ is speed normalized to solar wind speed.

the crosswind and downwind directions, respectively. These figures clearly manifest how variable the spectra are with respect to spectral intensity, slope and peak position when studied over the whole solar cycle.

The changes in the positions of the maximum of the slope of the PUI distribution function with respect to $w$ reflect changes in the heliocentric distance of the regions of maximum of neutral helium distribution. One can verify this by checking the correlation between the position of the slope maxima in the above mentioned figures with the positions of density maxima, presented in Fig. 3. The maximum of density is closest to the Sun during solar minimum and the maximum slope for this epoch occurs for lowest $w$. This effect can also be observed in Figs. 20 and 21, where slopes' maxima are clearly shifting towards higher $w$ values from solar minimum to maximum.

With careful modelling, the position of density maximum for a specific offset angle $\theta$ can be used as tracer of the net loss rate of helium inside $1 \mathrm{AU}$, since the position of density maximum is correlated with this parameter. But the net loss rate is a sum of (appropriately averaged) EUV and electron ionization rates, and the EUV ionization rate is known from independent observations. Hence, the position of the maximum slope of the $\mathrm{He}^{+}$PUI distribution function can yield some insight on the behaviour of solar wind electrons inside $1 \mathrm{AU}$ (from observations performed at $1 \mathrm{AU}$, as by ACE), including its evolution during the solar cycle, which currently is not well understood. 


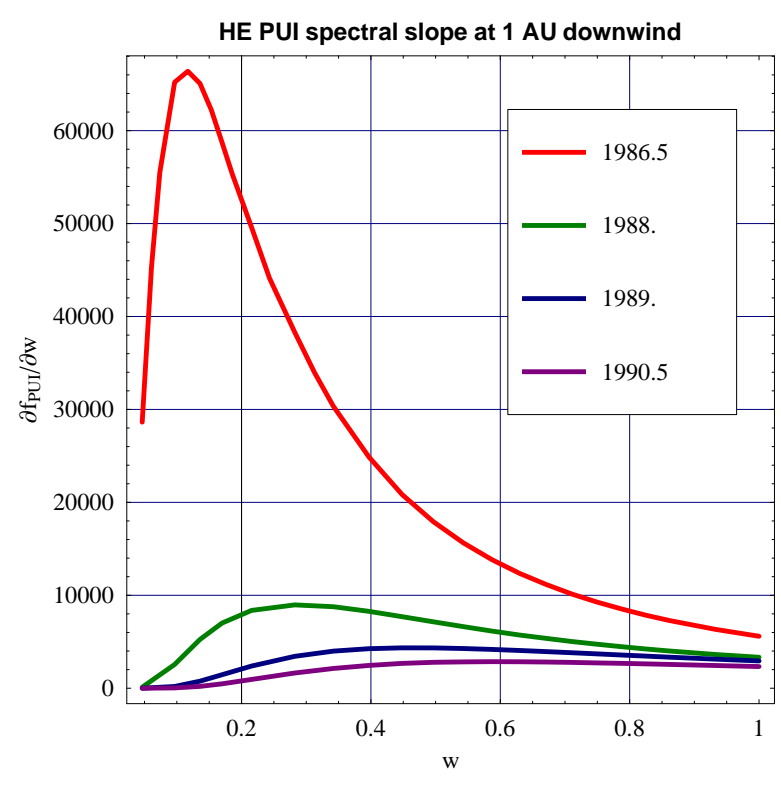

Fig. 21. Slopes of $f_{\text {PUI }}$ at four different phases of the solar cycle at $1 \mathrm{AU}$, downwind position; $w=v / V$ is speed normalized to solar wind speed.

\section{Discussion: Time dependence vs further complica- tions in interpretation of PUI observations}

The theoretically revealed aspects discussed in the former subsection should be taken as a serious hint to keep in mind when quantitative evaluations and interpretations of observed PUI distribution functions are the focus. A well-founded interpretation is not feasible without taking into account the solar cycle variability of the spectra. Further complications in the problem of interpreting the observed PUI spectra may arise due to the fact that the distribution function taken from Vasyliunas and Siscoe (1976), given by Eq. (11) and used here, is not fully correct due to existing pitch-angle anisotropies with differential PUI convection speeds and due to energy diffusion processes, as discussed in papers, e.g. by Isenberg (1997); Schwadron (1998); Isenberg and Lee (1998); Chalov and Fahr (1999a,b, 2000a,b, 2002).

The phase-space transport of interplanetary PUIs is a quite complicated problem with many different aspects. Several of these aspects have been addressed in some recent papers presenting theoretical results concerning the specifics of the phase-space behavior of pickup ions after their injection into the supersonic solar wind. Pickup ion spectra were obtained and revealed detailed spectral properties that could well be compared with observational data. Hereby effects of convection, adiabatic deceleration, pitch-angle diffusion, and even of energy diffusion could clearly be identified (see Gloeckler, 1999, 2003). It also became evident in the data that specific spectral differences between pickup spectra in slow solar wind streams and in fast solar wind streams can be found. For instance, the high-energy tails of pickup spectra beyond the injection energy were less pronounced in fast solar wind compared to slow streams (see Gloeckler et al., 2000). Quantities like the variable Alfvén velocities, turbulence levels, and the exposure times obviously do play important roles here as was demonstrated by Chalov and Fahr (2002).

Interesting as an observational basis of such PUI studies are pickup ion spectral observations obtained at moderate solar distances, i.e. inside $5 \mathrm{AU}$. In this heliospheric region many high-quality spectral PUI observations have been carried out with plasma analyzers on board of AMPTE, ULYSSES, SOHO, and ACE. The PUI spectra presented by Schwadron et al. (1996); Möbius et al. $(1998,1999)$ or Gloeckler (1996) and Gloeckler and Geiss (2001) are of very high quality with high statistical significance levels of the spectral fluxes up to energies of $10 \mathrm{keV} / \mathrm{amu}$. These observational data can indeed serve as a basis to control the quality and physical correctness of PUI transport theories applied to calculate PUI spectra.

In a recent paper by Chalov and Fahr (2000b), $\mathrm{He}^{+}$PUI spectra obtained by Möbius et al. (1999) were taken as a basis for comparisons with corresponding theoretical results. As was shown in this paper, the claim made by Möbius et al. (1999) that they can detect a signature of the actual injection velocity of $\mathrm{He}^{+}$PUIs in their data could in fact be supported by theoretical results, even though the complicated PUI phase-space transport under unfavorable solar wind conditions hides this effect somewhat. Particularly interesting in this respect was the predicted fact that typically different $\mathrm{He}^{+}$PUI spectra should result under conditions of fast or slow solar winds, respectively. At high solar wind velocities higher degrees of pitch-angle anisotropies and less pronounced high-energy tails of the spectra should occur. The deviations of the actual distribution functions from the form given by Eq. (11) will thus become non-negligibly large. The latter prediction in fact was observed in many PUI measurements, as clearly stated in the papers by Klecker et al. (2001) and Fisk et al. (2000). As was shown by Chalov and Fahr (2000b), these deviations are not only due to different exposure times for stochastic PUI scattering processes to operate, but also and even more to different conditions for MHD turbulence levels and Alfvén speeds. Hence, there are a number of important factors in addition to the time-dependent effects discussed here that need to be taken into account when analyzing PUI data.

Another issue that needs to be taken into account in the interpretation of $\mathrm{He}^{+}$PUI measurements is the interaction of interstellar $\mathrm{H}$ and $\mathrm{He}$ with solar wind alpha particles, which may lead to noticeable enhancement of the abundance of $\mathrm{He}^{+}$ions of solar wind characteristics at heliocentric distances typical for the Ulysses mission. The systematic enhancement may reach about an order of magnitude over the expected coronal abundance.

\section{Summary}

1. The distribution of interstellar neutral $\mathrm{He}$ atoms and fluxes of $\mathrm{He}^{+}$pickup ions are significantly affected by 
solar cycle effects (mainly by strong variations of the helium photoionization rate).

The deviations of helium density from the solar cycle mean in the regions penetrated by Ulysses are of the order of $10 \%$ and thus are very difficult to detect directly. However, the deviations from the mean of the $\mathrm{He}^{+}$pickup flux in this region are of the order of 50\% and basically can be detected by SWICS. The deviations of helium density rapidly increase with the decrease in heliocentric distance, and they exceed a factor of 1.5 at $0.5 \mathrm{AU}$ upwind and crosswind and at $4 \mathrm{AU}$ in the cone.

Variations of helium density caused by a modulation of the slowly variable photoionization rate by a 3\% 1-year periodic fluctuation (as due to charge exchange of neutral helium with solar wind particles) is noticeable only inside $0.3 \mathrm{AU}$ in the cone and $0.1 \mathrm{AU}$ elsewhere.

2. A pronounced "helium cone" structure, resulting from gravitational focusing of the inflowing interstellar $\mathrm{He}$ atoms, is clearly visible in the inner heliosphere (in neutral and pickup fluxes) during all phases of the solar cycle.

The downwind He density enhancement over the level of unperturbed He LISM density may vary at $1 \mathrm{AU}$ from $\sim 2$ (near solar maximum) to $\sim 7$ (around solar minimum).

The expected $\mathrm{He}^{+}$pickup ion fluxes in the downwind region are by a factor of $\sim 3-8$ higher than in the upwind hemisphere.

3. In the innermost regions $(r \sim 1 \mathrm{AU}$ upwind/crosswind; $r<3$ AU downwind) $\mathrm{He}^{+}$pickup production rates and related fluxes are anticorrelated with the solar activity. At larger distances (beyond $\sim 10-20$ AU their deviations from the mean follow the variability of the helium photoionization rate.

4. The position of maximum slope of the PUI distribution function with respect to $w=v / V$ corresponds to the heliocentric distance at which the maximum of helium density occurs at a particular time. This distance varies during the solar cycle and for each specific offset angle is correlated with the helium loss rate. Hence, the position of the maximum slope of $\mathrm{He}^{+}$PUI distribution function can be used as a tracer of the helium loss rate and its evolution during the solar cycle. Combined with an independent information on the EUV ionizing flux, this could allow one to determine the contribution of electron impact ionization to the net helium loss rate and thus give insight to the poorly known behaviour of solar wind electron parameters inside $1 \mathrm{AU}$.

Acknowledgements. H.J.F. and M.B. salute the memory of Dr. Daniel Ruciński, our good friend and the first author of this paper, who unexpectedly passed away in February 2002.

They are also indebted to the referees whose extremely useful and relevant reports by far exceeded the level of assistance for the authors that is usually expected.
D.R. and M.B. gratefully acknowledge hospitality of the Institut für Astrophysik Bonn (AIUB), where a major portion of this work was carried out. This research was supported by the Polish State Committee for Scientific Research grant 2P03C 00519 and by the DFG/PAS Cooperative Project 436 POL 113/80/0.

Topical R. Forsyth thanks A. Bogdanor and N. A. Schwadron for their help in evaluating this paper.

\section{References}

Bertaux, J.-L., Lallement, R., Kurt, V. G., and Mironova, E. N.: Characteristics of the local interstellar hydrogen determined from Prognoz 5 and 6 interplanetary Lyman- $\alpha$ profile measurements with a hydrogen absorption cell, Astron. Astrophys., 150, 1-20, 1985.

Blum, P., Pfleiderer, J., and Wulf-Mathies, C.: Neutral gases of interstellar origin in interplanetary space, Planet. Space Sci., 23, 93-105, 1975.

Burgin, M. S., Kurt, V. G., Mironova, E. N., Prokhorenko, V. I., Ejsmont, N. A., Bertaux, J. L., and Dalaudier, F.: Atomy gelija w mezhzwezdnoy srede. I. Nabludeniya rasseyanya ultrafioletovogo izlucheniya w linyakh H 1216 A i He I 584 A s ISZ "Progonoz 5" i "Prognoz 6", Kosm. Issled., 21, 83-94, 1983.

Chalov, S. V. and Fahr, H. J.: Signatures of the interplanetary helium cone reflected by pick-up ions, Solar Phys., 187, 123-144, 1999a.

Chalov, S. V. and Fahr, H. J.: Interplanetary pick-up ion acceleration. A study of anisotropic phase-space function, Astrophys. Space Sci., 264, 509-525, 1999 b.

Chalov, S. V. and Fahr, H. J.: Pick-up ion acceleration at the termination shock and the post-shock pick-up ion energy distribution, Astron. Astrophys., 360, 381-390, 2000a.

Chalov, S. V. and Fahr, H. J.: Keplerian injection velocities reflected in helium pick-up ion spectra, Astron. Astrophys., 363, L21-L24, 2000b.

Chalov, S. V. and Fahr, H. J.: Different solar wind types reflected in pick-up ion spectral signatures, Astron. Astrophys., 384, 299302, 2002.

Chassefière, E., Bertaux, J. L., Lallement, R., and Kurt, V. G.: Atomic hydrogen and helium densities of the interstellar medium measured in the vicinity of the Sun, Astron. Astrophys., 160, 229-242, 1986.

Chassefière, E., Bertaux, J. L., Lallement, R., Sandel, B. R., and Broadfoot, L.: A new estimate of interstellar helium parameters from measurements of Voyager 1/2 ultraviolet spectrometers, Astron. Astrophys., 199, 304-311, 1988.

Costa, J., Lallement, R., Quémerais, E., Bertaux, J. L., Kyrölä, E., and Schmidt, W.: Heliospheric interstellar $\mathrm{H}$ temperature from SOHO/SWAN H cell data, Astron. Astrophys., 349, 660-672, 1999.

Dalaudier, F., Bertaux, J. L., Kurt, V. G., and Mironova, E. N.: Characteristics of interstellar helium observed with Prognoz 6 58.4nm photometers, Astron. Astrophys., 134, 171-184, 1984.

Fahr, H., Fichtner, H., and Scherer, H.: Extreme-ultraviolet diagnostics of pick-up ions in regions close to the solar corona. I. Feasibility of new observations, Astron. Astrophys., 335, 724732, 1998.

Fahr, H. J.: The interplanetary hydrogen cone and its solar cycle variations, Astron. Astrophys., 14, 263-274, 1971.

Fahr, H. J.: Interstellar hydrogen subject to a net repulsive solar force field, Astron. Astrophys., 77, 101-109, 1979. 
Fahr, H. J., Lay, G., and Blum, P. W.: Planetary cones of focused interstellar gas, Astron. Astrophys., 52, 363-372, 1976.

Fahr, H. J., Ruciński, D., and Nass, H. U.: Modelling of the interplanetary helium EUV-resonance glow for time-dependent solar radiation conditions, Ann. Geophysicae, 5A, 255-264, 1987.

Fahr, H. J., Osterbart, R., and Ruciński, D.: The effect of the heliospheric filtration on the distant Lyman-Alpha glow and the pickup proton fluxes, Astron. Astrophys., 274, 612-620, 1993.

Feldman, W. C., Lange, J. J., and Scherb, F.: in: Solar Wind, NASA SP 308, p. 684, NASA, 1972.

Fisk, L. A., Gloeckler, G., Zurbuchen, T. H., and Schwadron, N. A.: Ubiquitous statistical acceleration in the solar wind, in ACE Symposium 2000 - Acceleration and transport of energetic particles, (Ed) Mewaldt, R. A., no. 1-56396-951-3 in CP-528, pp. 229-233, American Institute of Physics, 2000.

Flynn, B., Vallerga, J., Dalaudier, F., and Gladstone, G. R.: EUVE measurement of the local interstellar wind and geocorona via resonance scattering of solar He I 584-A line emission, J. Geophys. Res., 103, 6483-6494, 1998.

Freeman, J., Paresce, F., Bowyer, S., and Lampton, M.: Observations of interstellar helium with a gas absorption cell: limits on the bulk velocity of the interstellar medium, Astrophys. J., 208, 747-752, 1976.

Freeman, J., Paresce, F., and Bowyer, S.: The local interstellar helium density: corrected, Astrophys. J., 231, L37-L39, 1979.

Gloeckler, G.: The abundance of atomic ${ }^{1} \mathrm{H},{ }^{4} \mathrm{He}$ and ${ }^{3} \mathrm{He}$ in the local interstellar cloud from pickup ion observations with SWICS on Ulysses, Space Sci. Rev., 78, 335-346, 1996.

Gloeckler, G.: Observation of Injection and Pre-Acceleration Processes in the Slow Solar Wind, Space Sci. Rev., 89, 91-104, 1999.

Gloeckler, G.: Ubiquitous suprathermal tails on the solar wind and pickup ion distributions, in: Solar Wind 10, 2003.

Gloeckler, G. and Geiss, J.: Heliospheric and Interstellar Phenomena Deduced From Pickup ion Observations, Space Sci. Rev., 97, 169-181, 2001.

Gloeckler, G., Jokipii, J. R., Giacalone, J., and Geiss, J.: Concentration of interstellar pickup $\mathrm{H}^{+}$and $\mathrm{He}^{+}$in the solar wind, Geophys. Res. Lett., 21, 1565-1568, 1994.

Gloeckler, G., Fisk, L. A., Zurbuchen, T. H., and Schwadron, N. A.: Sources, Injection and Acceleration of Heliospheric Ion Populations, in: AIP Conf. Proc. 528: Acceleration and Transport of Energetic Particles Observed in the Heliosphere, p. 221, 2000.

Isenberg, P. A.: A hemispherical model of anisotropic interstellar pickup ions, J. Geophys. Res., 102, 4719-4724, 1997.

Isenberg, P. A. and Lee, M. A.: Effects of time-dependent photoionization on interstellar pickup ions, J. Geophys. Res., 100, 17 053-17 058, 1995.

Isenberg, P. A. and Lee, M. A.: Transport of interstellar pickup ions on bent flux tubes, J. Geophys. Res., 103, 12 037-12 047, 1998.

Judge, D. L., McMullin, D. R., Ogawa, H. S., Hovestadt, D., Klecker, B., Hilchenbach, M., Möbius, E., Canfield, L. R., Vest, R. E., Watts, R., Tarrio, C., Kuehne, M., and Wurz, P.: First Solar EUV Irradiances Obtained from SOHO by the CELIAS/SEM, Solar Phys., 177, 161-173, 1998.

Klecker, B., Bogdanov, A. T., Hilchenbach, M., Galvin, A. T., Möbius, E., Ipavich, F. M., and Bochsler, P.: On the variability of suprathermal pickup $\mathrm{He}^{+}$at $1 \mathrm{AU}$, in: The outer heliosphere: the next frontiers, (Eds) K. Scherer, H. Fichtner, H. J. Fahr, and E. Marsch, COSPAR Colloquia Series Vol. 11, p. 229, Elsevier, Pergamon, 2001.

Kurt, V. G., Mironova, E. N., Bertaux, J. L., and Dalaudier,
F.: Atomy gelija w mezhzvezdnoj i mezhplanetarnoj srede. II. Opredelenije naprawlenija dwizhenija mezhzwezdnoj sredy otnositelno Solnca, Kosm. Issled, 22, 97-102, 1984a.

Kurt, V. G., Mironova, E. N., Bertaux, J. L., and Dalaudier, F.: Atomy gelija w mezhzvezdnoj i mezhplanetarnoj srede. III. Temperatura i skorost mezhzwezdnogo vetra, Kosm. Issled, 22, 225 237, 1984b.

Lallement, R.: Global structure of the heliosphere: Optical observations, in: Solar Wind Nine, AIP Conference Proceedings 471, pp. 205-210, American Institute of Physics, 1999.

Lallement, R. and Bertin, P.: Northern hemisphere observations of nearby interstellar gas: Possible detection of the local cloud, Astron. Astrophys., 266, 479-485, 1992.

Michels, J. G., Raymond, J. C., Bertaux, J. L., Quémerais, E., Lallement, R., Ko, Y.-K., Spadaro, D., Gardner, L. D., Giordano, S., O’Neal, R., Fineschi, S., Kohl, J. L., Benna, C., Ciaravella, A., Romoli, M., and Judge, D.: The Helium Focusing Cone of the Local Interstellar Medium Close to the Sun, Astrophys. J., 568, 385-395, 2002.

Möbius, E., Hovestadt, D., Klecker, B., Scholer, M., Gloeckler, G., and Ipavich, F. M.: Direct observation of $\mathrm{He}^{+}$pickup ions of interstellar origin in the solar wind, Nature, 318, 426-429, 1985

Möbius, E., Ruciński, D., Hovestadt, D., and Klecker, B.: The helium parameters of the Very Local Interstellar Medium as derived from the distribution of $\mathrm{He}^{+}$pickup ions of interstellar origin in the solar wind, Astron. Astrophys., 304, 505-519, 1995.

Möbius, E., Ruciński, D., Isenberg, P. A., and Lee, M. A.: Determination of interstellar pickup ion distribution in the solar wind with SOHO and Cluster, Ann. Geophysicae, 14, 492-496, 1996.

Möbius, E., Ruciński, D., Lee, M. A., and Isenberg, P. A.: Decreases in the antisunward flux of interstellar pickup $\mathrm{He}^{+}$associated with radial interplanetary magnetic field, J. Geophys. Res., 103, 257-265, 1998.

Möbius, E., Litvinenko, Y., Grünwaldt, H., Aellig, M. R., Bogdanov, A., Ipavich, F. M., Bochsler, P., Hilchenbach, M., Judge, D., Klecker, B., Lee, M. A., and Ogawa, H.: Direct evidence of the interstellar gas flow velocity in the pickup ion cutoff as observed with SOHO CELIAS CTOF, Geophys. Res. Lett., 26, 3181-3184, 1999.

Ogawa, H. S., Phillips, E., and Judge, D. L.: Direct measurements of the helium photoionization rate from sounding rockets, J. Geophys. Res., 102, 11 557-11 566, 1997.

Ogawa, H. S., Judge, D. L., McMullin, D. R., and Gangopadhyay, P.: First-year continuous solar EUV irradiance from SOHO by the CELIAS/SEM during 1996 solar minimum, J. Geophys. Res., 103, 1-6, 1998.

Osterbart, R. and Fahr, H. J.: A Boltzmann-kinetic approach to describe entrance of neutral interstellar hydrogen into the heliosphere, Astron. Astrophys., 264, 260-269, 1992.

Paresce, F., Bowyer, S., and Kumar, S.: Observations of He I 584 A nighttime radiation; evidence for an interstellar source of neutral helium, Astrophys. J., 187, 633-639, 1974a.

Paresce, F., Bowyer, S., and Kumar, S.: Further evidence for an interstellar source of nighttime He I 584 A radiation, Astrophys. J., 188, L71-L73, 1974b.

Paresce, F., Fahr, H., and Lay, G.: A search for interplanetary He II, 304-A emission, J. Geophys. Res., 86, 10 038-10 048, 1981.

Ruciński, D. and Bzowski, M.: Modulation of interplanetary hydrogen distribution during the solar cycle, Astron. Astrophys., 296, 248-263, 1995.

Ruciński, D. and Fahr, H. J.: Nonthermal ions of interstellar origin at different solar wind conditions, Ann. Geophysicae, 9, 102- 
110, 1991.

Ruciński, D., Fahr, H. J., and Grzędzielski, S.: The production of pick-up ion species in the heliosphere and their convection towards the three-dimensional termination shock, Planet. Space Sci., 41, 773-783, 1993.

Ruciński, D., Cummings, A. C., Gloeckler, G., Lazarus, A. J., Möbius, E., and Witte, M.: Ionization processes in the heliosphere - rates and methods of their determination, Space Sci. Rev., 78, 73-84, 1996.

Ruciński, D., Bzowski, M., and Fahr, H. J.: Minor helium components co-moving with the solar wind, Astron. Astrophys., 334, 337-354, 1998.

Schwadron, N. A.: A model for pickup ion transport in the heliosphere in the limit of uniform hemispheric distributions, J. Geophys. Res., 103, 20 643-20 649, 1998.

Schwadron, N. A., Fisk, L. A., and Gloeckler, G.: Statistical acceleration of interstellar pickup ions in corotating interaction regions, Geophys. Res. Lett., 23, 2871-2874, 1996.

Vasyliunas, V. and Siscoe, G.: On the flux and the energy spectrum of interstellar ions in the solar wind, J. Geophys. Res., 81, 1247-
1252, 1976.

Weller, C. S. and Meier, R. R.: Observations of helium in the interplanetary /interstellar wind: the solar - wake effect, Astrophys. J., 193, 471-476, 1974.

Weller, C. S. and Meier, R. R.: Analysis of the helium component of the Local Interstellar Medium, Astrophys. J., 227, 816-823, 1979.

Weller, C. S. and Meier, R. R.: Characteristics of the helium component of the Local Interstellar Medium, Astrophys. J., 246, 816823, 1981.

Witte, M., Banaszkiewicz, M., Rosenbauer, H., and McMullin, D. R.: Kinetic parameters of the interstellar helium: updated results from the Ulysses/GAS instrument, Adv. Space Res., in press 2003.

Witte, M., Rosenbauer, H., Banaszkiewicz, M., and Fahr, H. J.: Recent results on the parameters of the interstellar helium from the Ulysses/GAS experiment, Space Sci. Rev., 78, 289-296, 1996.

Wu, F. M. and Judge, D. L.: Temperature and velocity of the interplanetary gases along solar radii, Astrophys. J., 231, 594-605, 1979. 